\title{
Current Trend in Fabrication of Complex Morphologically Tunable Superhydrophobic Nano Scale Surfaces
}

\author{
Ali T Abdulhussein ${ }^{\mathrm{a}}$, Ganesh K Kannarpady ${ }^{\mathrm{a}^{*}}$, Andrew B Wright ${ }^{\mathrm{b}}$, Anindya Ghosh ${ }^{\mathrm{c}}$, Alexandru S Biris $\mathrm{a}^{\mathrm{a}^{* *}}$ \\ ${ }^{\text {a }}$ Center for Integrative Nanotechnology Sciences, University of Arkansas at Little Rock \\ 2801 South University Avenue, Little Rock, AR, 72204 \\ ${ }^{\mathrm{b}}$ Department of Systems Engineering, University of Arkansas at Little Rock \\ 2801 South University Avenue, Little Rock, 72204 \\ ${ }^{c}$ Department of Chemistry, University of Arkansas at Little Rock \\ 2801 South University Avenue, Little Rock, AR 72204, USA \\ Corresponding authors:*gkkannarpady@ualr.edu; ** asbiris@ualr.edu
}

\begin{abstract}
Superhydrophobic surfaces are found in nature and possess several fascinating properties, including the ability to self-clean. A typical superhydrophobic surface has micro/nanostructure roughness and low surface energy, which combine to give it its unusual anti-wetting properties. Because of their unique capabilities, these surfaces have interested scientists in research and industry fields for years. In recent decades, researchers have developed a number of synthetic methods for producing novel superhydrophobic surfaces that mimic natural surfaces. These synthetic surfaces have been widely applied on different types of substrates for potential widespread, practical applications. This review article focuses on these advances in fabricating manmade superhydrophobic surfaces.
\end{abstract}

Keywords: Superhydrophobic surfaces, Self-cleaning, Nanostructure roughness, Surface energy, Anti-wetting.

\section{Introduction}

Many biomimetic materials and surfaces interest scientists and engineers. The term "biomimetic" refers to a design, adaption, or derivation taken from nature [1,2]. Nano/microstructures have been revealed in many types of animals and plants, including the lotus leaf, butterfly wing, water strider leg, moth eye, and shark scales-all of which exhibit 
great hydrophobicity/superhydrophobicity, or non-wetting properties. For example, the lotus leaf's self-cleaning characteristics are directly linked to its hierarchical structure. In fact, its superhydrophobic properties result from a combination of micro/nanostructures and low surface energy.

Recently, scientists have discovered many artificial techniques for designing superhydrophobic surfaces that mimic naturally self-cleaning surfaces [3-14]. Surface water wettability is determined by the contact angle formed between a drop of water and the surface that it rests upon. Superhydrophobic surfaces are typically described as follows: the water contact angle (CA) must be greater than $150^{\circ}$, and a water droplet must be capable of rolling off the surface at a surface tilting angle of less than $10^{\circ}$, usually referred to as the sliding angle. Superhydrophobic surfaces offer special properties such as water repellency, anti-sticking, and anti-contamination because these surfaces have a water contact angle larger than $150^{\circ}$, in addition to considerable roughness. As a result, water droplets are apt to roll off such surfaces. Significant scientific effort has been dedicated to fabricating superhydrophobic surfaces with surface structure roughness created by micro/nanostructures that exhibit superhydrophobicity. Some fabrication methods are simple and cost effective, but many require numerous procedures or specialized equipment.

Several review articles have been published by various researchers on different aspects of superhydrophobic surfaces. Superhydrophobicity and its implications in mitigating marine fouling were reviewed by Genzer and Efimenkoin [15]. They discussed recent developments in fabricating cost-effective superhydrophobic surfaces by tailoring chemical/physical properties; they also discussed the surfaces' responsiveness as marine anti-fouling coatings. Stratakis and Zorba [16] reviewed artificial nanostructured surfaces produced by mimicking natural superhydrophobic surfaces. Their detailed review focused on different approaches employed to fabricate such nature-inspired artificial surfaces and their applications. Gue et al. [17] reviewed some recently published studies on both natural and biomimetic superhydrophobic surfaces, including different smart routes for fabricating superhydrophobic surfaces and their applications. Celia et al. [18] reviewed several techniques to produce superhydrophobic surfaces with low contact angle hysteresis, reentrant structures for omniphobic applications, etc. 
In contrast to these past reviews, this review will present the fundamentals of superhydrophobicity and an overview of synthetic superhydrohobic developments. The first section discusses the theoretical principles behind the wetting of a solid surface by a liquid, as well as a brief review of the most common superhydrophobic surfaces in nature. Section two provides an overview of different methods for creating superhydrophobic surfaces, including the materials, the fabrication methods, the micro/nanostructures that have been created for the preparation of superhydrophobic surfaces, and their applications in industry.

\subsection{Superhydrophobicity Principles}

Water contact angle $(\theta)$ is the major measure of surface hydrophobicity. This angle is made when a water droplet is laid on a surface. The contact angle can be described as the tangent angle of the interface between the liquid and vapor at the three phases of liquid-vapor-solid described as the contact line (CL) (Fig. 1).

For ideally flat and smooth, as well as chemically homogenous, surfaces, the contact angle is only described by Young`s equation (Fig. 1) [19]:

$\cos \theta=\frac{\left(\gamma_{s v}-\gamma_{s l}\right)}{\gamma_{l v}}$

where $\gamma_{s v}, \gamma_{s l}$ and $\gamma_{l v}$ are the interfacial tensions between solid-vapor, solid-liquid, and liquidvapor, respectively.

A surface is termed hydrophilic if the contact angle is below $90^{\circ}$, hydrophobic if it is larger than $90^{\circ}$, and superhydrophobic if it is larger than $150^{\circ}$.

If the wetting is performed on rough and solid surfaces, though, it becomes more complicated. In fact, two models have been developed to demonstrate the behavior of a droplet resting on rough surfaces. Surface roughness and its impact on contact angle were described by the Wenzel and Cassie-Baxter equations [20,21].

The Wenzel model shows that the droplet may completely penetrate the rough asperities, and, as a consequence, the contact area of the surface is increased (Fig. 2) [20]. According to the Wenzel model, the contact angle over rough surfaces is described by this equation:

$\cos \theta_{W}=\frac{r\left(\gamma_{s v}-\gamma_{s l}\right)}{\gamma_{l v}}=\mathrm{r} \cos \theta$ 
where $\theta_{W}$ is the contact angle on a rough surface, $\theta$ is the contact angle according to Young`s model on a smooth surface, and $\mathrm{r}$ is the roughness factor of the surface:

$$
\mathrm{r}=\frac{\text { actual surface area }}{\text { planar area }}
$$

when $r=1$ the surface is ideally smooth, and the surface is rough if $r$ is greater than 1 . The Wenzel equation emphasizes that wetting is promoted by surface roughness if $\theta$ is $<90^{\circ}$, and the wetting is decreased if $\theta$ is $>90^{\circ}$.

The Cassie-Baxter state [21] is also referred to as the heterogonous state. In this state, the asperities beneath the droplet are covered by vapor, and the liquid does not enter the surface grooves (Fig. 3). In this case, the liquid-surface interface is an interface comprised of two phases, a liquid-solid interface, $f_{1}$, and a liquid-vapor interface, $f_{2}$. According to the Cassie-Baxter model, the apparent contact angle is the sum of all contributions of different phases as shown in the following equation:

$\cos \theta_{C B=} f_{1} \cos \theta_{1}+f_{2} \cos \theta_{2}$

where $f_{1}$ and $f_{2}$ are the surface fractions for both phase 1 and 2, respectively; $\theta_{1}$ and $\theta_{2}$ are the contact angles for phase 1 and phase 2 .

This equation can also be applied when no roughness exists. If one of these surfaces is the interface between the vapor and liquid, $f$ is the fraction of a solid and is described as the solid surface fraction, which is wetted by the liquid. Thus, $\left(1-f_{1}\right)$ is the fraction of vapor. The resulting contact angle can be obtained with $\theta$ equal to $180^{\circ}$ for vapor using this equation:

$\cos \theta_{C B}=f \cos \theta+(1-f) \cos 180^{\circ}=f \cos \theta+f-1$

The surface fraction, $f$, is between (0-1); the surface is fully wetted when $f=1$, and the liquid droplet is never in contact with the surface when $f=0$, which is the same as flat surface behavior. If a droplet is in the Cassie-Baxter state, the droplet can be rolled much more easily onto the surface due to the small contact area between the solid surface and the droplet. Generally, in the Cassie-Baxter state a liquid droplet on a rough surface exhibits a lower sliding angle value than in the Wenzel state [22]. For many surfaces, the transition from Cassie to Wenzel states has been observed under the conditions of vibration, high impact, or when a 
droplet is exposed to pressure; however, a reversible transition from the Wenzel to the Cassie state may result from heating the substrate or applying vibration to a cold substrate (Fig. 4) [2328].

For understanding wetting on rough surfaces, Marmur [29] demonstrated that both the Wenzel and Cassie-Baxter equations are essential but not sufficient to describe wetting equilibrium. He formulated a complete set of equilibrium conditions of a droplet on a rough surface, emphasizing the relationship between the solid surface topography and the transition between Wenzel (homogenous) and Cassie-Baxter (heterogonous) wetting regimes.

The dynamics of water droplets on superhydrophobic surfaces can be predicted using the contact angle hysteresis (CAH). The water contact angle hysteresis is the difference between the advancing contact angle and the receding contact angle. It is one of the common approaches to representing liquid droplet motion on a surface. In general, a superhydrophobic surface has low contact angle hysteresis, meaning that a water droplet would roll off the surface with a slight tilt [30].

Water droplet bouncing is another significant phenomenon in wetting behavior. A droplet could stick, spread, or bounce when it hits a surface. In practical applications, superhydrophobic surfaces should maintain their capability to repel the penetrating droplets under dynamic conditions. Numerous researchers have considered the dynamic influences of droplets on superhydrophobic surfaces. The different modes of water droplet/surface impact (spreading, rebound, and splash) have been related to impact velocity, volume of droplets, and surface properties, as well as the physical properties of the liquid employed. Water droplet/surface contact time, bounce elasticity, and morphology of the droplet have also been studied [31-35].

\subsection{Superhydrophobic Surfaces in Nature}

Many plants and animals possess natural superhydrophobic surfaces [7,36-39]. One of the best known examples are lotus leaves, Nelumbo nucifera, which have self-cleaning properties - the ability to remove dust and other particles due to the rolling away of water droplets on the leaves' surface (Fig. 5 A). Neinhis and Barthlott [40] reported that lotus leaves are composed of epidermal cells that form papilla with microbumps, which are covered with epicuticular wax; these nanohair-like structures allow a droplet to roll on the surface, making the leaves self-cleaning [41]. The static contact angle of a lotus leaf was determined to be $164^{\circ}$ with 
a $3^{\circ}$ contact angle hysteresis $[1,2,42]$. These findings encouraged scientists to mimic the lotus leaves' structure to design synthetic surfaces with self-cleaning properties.

Like the lotus leaf, taro leaves have a micro/nano surface topography. The surface structure of a taro leaf is characterized by uniformly disseminated micro-elliptic prominences (Fig. 5 B). It has been observed by scanning electron microscope (SEM) that nanometer pins are distributed across the entire surface, leading to a superhydrophobic surface with a higher contact angle and very low sliding angle [43]. On the other hand, a few plants with floating leaves have very structured surfaces, especially Salvinia molesta leaves, whose surfaces include dense hairs with multicellular cells. These cells resemble microscopic "eggbeaters" with hydrophilic patches at the tips of the hairs, presumably to stabilize the air film against pressure fluctuation (Fig. 5 C) [44].

The Morpho aega, which is a neotropical butterfly observed in some regions in South America, employs the superhydrophobic effect by a natural mechanism in watery environments. In moist environments, a water droplet can roll off the surface along the radial outward (RO) direction of the butterfly's body, demonstrating superhydrophobicity. Zheng et al. [45] used a SEM to observe that the wings' surfaces were coated with overlapping scales as periodic arrangements that form a hierarchy over the radial outward (RO) direction. Figure 6 A reveals that the microscales are composed of fine lamella stacking strips that accumulate stepwise over the RO direction.

A water strider has the ability to walk, jump, and slide on water, utilizing its waterresistant legs. The legs are water-resistant because they are covered with hydrophobic microhairs that have nano-grooves (Fig. 6 B). Air is trapped in the region between the micro- and nanostructures on the hairs, making the legs water-repellant $[5,46]$.

The back of the Stenocara beetle shows superhydrophobic properties similar to those of lotus leaves. Stenocara elytra are coated with a set of semi-random bumps approximately 0.5-1.5 $\mathrm{mm}$ apart, and each bump has a diameter of $0.5 \mathrm{~mm}$ (Fig. 6 C) [39,47]. SEM images reveal that the summits of those bumps are smooth and without covering, while the microtexture of their sloping sides is covered with a waxy substance. The microtexture is composed of flattened hemispheres having a diameter of $10 \mathrm{~mm}$ constructed in a uniform hexagonal arrangement [47]. 
A model surface for self-cleaning and superolephobicity (oil-repellency) is found in fish, which are known to have the ability to protect themselves from oil pollution even though they are wetted by water [48]. The skin of a shark — a natural model for a low drag surface — is coated by dermal denticles, tiny individual tooth-like scales, supported with longitudinal grooves aligned parallel to the water's local flow direction (Fig. 7 A) [49]. The grooved scales decrease the formation of vortices on a smooth surface, leading to water moving efficiently above their surfaces. The water that surrounds these complex structures prevents marine fouling and resists the adhesion and aggregation of marine organisms such as bacteria and algae [2,48,50,51]. A gecko, which has the ability to climb vertical surfaces [52,53], has millions of keratinous hairs or setae on its feet (Fig. 7 B). Each seta is about 30-130 $\mu \mathrm{m}$ long and contains hundreds of $200 \mathrm{~nm}$ spatulae that form hierarchical morphologies that make intimate contact with various surface profiles $[4,53,54]$.

Additional examples of multifaceted structures in nature include the superhydrophobicity and anti-reflection functions of cicada wings [55,56]; the superhydrophobicity, anti-reflection, and anti-fogging of mosquito eyes [18,57,58]; the structural color, and superhydrophobicity of peacock feather [59]; and the anti-reflection and superhydrophobicity properties of moth eyes $[39,55]$. Clearly, nature offers a great number of biological materials with multiscale, multifunctional structures that play a major role in achieving functional integrity [6].

\section{Methods for Fabricating Superhydrophobic Surfaces}

The Wenzel and Cassie models indicate that the roughness of a surface is integral in promoting both hydrophilic and hydrophobic surfaces. Many superhydrophobic surfaces in nature have remarkable roughness and micro/nanostructures. Scientists have utilized various methods to mimic nature's inherently superhydrophobic surfaces. The techniques employed for superhydrophobic surface fabrication can be classified into the "top-down" and "bottom-up" methods. Top-down approaches involve lithography, plasma treatment of the surfaces, and template-based techniques. Bottom-up methods include chemical deposition, layer-by-layer deposition, and colloidal assemblies. There are also techniques that combine the bottom-up and top-down methods, such as the casting of a polymer solution and phase separation.

To accomplish such complex responses at the interface between a solid surface and water droplets, the surface must meet a multitude of technical requirements. For example, the surface 
has to present multidimensional nano/microscale morphologies and have tunable chemical characteristics. In this section, we will review the most recently published articles that discussed the successful synthesis of superhydrophobic surfaces.

\subsection{Lithography Methods}

Lithography is a method for transferring a computer-designed pattern onto a substrate surface $[60,61]$ and is one of the most widely employed methods of making superhydrophobic surfaces. Lithography makes it possible to control surface morphology and encompasses many techniques: photolithography, soft lithography, electron beam lithography, nanoimprinting lithography, x-ray lithography, and colloidal lithography [61-66]. Li et al. [67] utilized direct laser interference lithography to fabricate highly ordered superhydrophobic structures with dual micro- and nanoscales on the silicon wafer surface. These superhydrophobic structures were made by controlling the interference process of a four-beam laser and employing hydrofluoric acid (HF) to remove the silica that formed during the process. The team used a variety of parameters for the duration of exposure, cleanout times, and the laser fluences to achieve the highest contact angle. An exposure of 60 seconds and immersion in a 5\% concentration of hydrofluoric acid resulted in a superhydrophobic surface with the optimum contact angle of $153.2^{\circ}$ (Fig. 8). Cleanout times, exposure duration, and laser fluence all affected the contact angle value (Fig. 9).

Kang et al. [68] successfully presented a simple and cheap method to fabricate superhydrophobic surfaces utilizing Marangoni instability. The method involves in situ photopolymerization (SCP) in a volatile solvent and covering the surface with porous Polydimethylsiloxane (PDMS) to produce a superhydrophobic surface with desired roughness of nano- and microstructures. The superhydrophobic surface was produced by a facile and simple process that included spreading, covering, and SCP without the need for fluorinated chemistry and complicated equipment. The porous PDMS cover worked effectively to create a superhydrophobic surface with a contact angle higher than $160^{\circ}$ (Fig. 10 A) due to its influence in the Marangoni convection of the coating fluid, which induced composition gradients at the same time. On the other hand, the concentration variations of ethanol resulted in surfaces ranging from hydrophilic to superhydrophobic. The ethanol concentration has a significant effect in determining the contact angle value (Fig. 10 B and C). This study also successfully demonstrated the application of prepared superhydrophobic surfaces in oil capture and transportation. 
Choi et al. [69] fabricated superhydrophobic surfaces with overhang textures using reverse nano-imprint lithography and ion reactive etching, simultaneously. A fluoroalkylsilane monolayer was deposited on the overhanging structures to decrease the surface energy. Moreover, four kinds of nano-patterns were independently embedded over the surface to promote many properties, such as oil-repellency, by modified reverse imprinting lithography.

\subsection{Wet/Dry Etching}

In general, etching means the removal of a material either via a chemical method using liquid etchants (wet etching) or a plasma process using plasma (dry etching). Wet chemical etching is an easily controllable approach compared to other techniques. Additionally, it is very suitable for commercial and industrial adoption of multi-material, large-scale, and hierarchical superhydrophobic functions [70-74]. Liao et al. [75] used a continuous chemical etching approach to obtain a superhydrophobic surface on aluminum foil. They obtained microstructures after immersing the aluminum foil in $\mathrm{CuCl}_{2}$ solution (Fig. 11. A a and c). The reversible hydrolysis equilibrium between the reaction of water molecules and $\mathrm{Cu}^{2+}$ ions produces a sufficient amount of $\mathrm{H}_{2}$ as well as heat, which changes the aqueous solution to "a boiling state" [76]. The $\mathrm{HCl}$ used for additional etching resulted in the hierarchal micro-nano binary morphology (Fig. 11. A b, d, and f). Hexadecyltrimethoxy silane was employed to anchor the surface of aluminum after the self-assembly reaction. This work resulted in a superhydrophobic aluminum surface with a contact angle of $161.9 \pm 0.5^{\circ}$ and a sliding angle of $6.8 \pm 1^{\circ}$ (Fig. $11 \mathrm{~B}$ ). Such superhydrophobic surfaces are able to mitigate freezing in glaze ice, making it well-suited for anti-icing applications.

Li et al. [77] fabricated superhydrophobic iron surfaces using chemical etching methods. The hierarchical structures were created on the surface of iron after immersion in $\mathrm{HCl}$ solution or galvanic replacement of silver nitrate $\left(\mathrm{AgNO}_{3}\right)$ solution for a specific period. The next hydrophobic modification using stearic acid produced superhydrophobic surfaces with high contact angles. An increase in the $\mathrm{HCl}$ concentration from $4 \mathrm{~mol} / \mathrm{L}$ to $8 \mathrm{~mol} / \mathrm{L}$ resulted in higher contact angle values ranging from $127^{\circ}$ to $152^{\circ}$. The $\mathrm{AgNO}_{3}$ concentration only slightly affected the contact angle value of the superhydrophobic iron surface, whereas the silver particles had a preferential growth direction resulting in the creation of silver dendritic crystals. In comparison with the untreated iron, the superhydrophobic iron surface exhibited excellent anti-icing properties. 
Barshilia and Gupta [78] successfully prepared a superhydrophobic surface on Polytetrafluoroethylene (PTFE/Teflon) by using the $\mathrm{Ar}+\mathrm{O}_{2}$ plasma-etching approach. Teflon, the trademark name of PTFE, is a fluoro polymer with great thermal and chemical stability. The surface morphology of PTFE samples showed 'leaf-like' micro-bumps after treatment with plasma using field emission scanning electron microscopy (FESEM) analysis. The samples of PTFE were treated with $\mathrm{Ar}+\mathrm{O}_{2}$ plasma etching for time periods ranging from 1-5 hours, categorized as TF1-TF5 (Fig. 12 A). The highest contact angle of $158^{\circ}$ was obtained at a treatment time of 4 hours. Surface profile measurements showed that the average surface roughness increased until the treatment time of 4 hours, which was considered the optimum treatment time (Fig. 12 B). There was not much variation in the chemical structure of the untreated and $\mathrm{Ar}+\mathrm{O}_{2}$ plasma-treated PTFE samples when they were chemically analyzed by micro-Raman and X-ray photoelectron spectroscopy. This study demonstrated that a change in the surface roughness is enough to fabricate superhydrophobic PTFE surfaces; consequently, they can be utilized in biotechnological, biomedical, and electrical insulation applications.

\subsection{Chemical Vapor Deposition (CVD)}

Chemical Vapor Deposition (CVD) is a technique in which a gas precursor is deposited on a heated substrate to form the desired solid film. Chemical reactions are included in this method. CVD can be employed to prepare a rough surface, make micro-/nanoparticles, transform nanorods into macrostructures, or deposit a thin hydrophobic film onto rough surfaces [17,7982]. Currently, CVD as a technique to deposit hydrophobic films is an area of great interest [17]. Singh et al. [83] reported the static and dynamic wetting properties of a 3D graphene foam coated with Teflon (Fig.13 A) that was utilized to fabricate a superhydrophobic structure. The foam was synthesized using template-directed CVD and consisted of a pore structure of many hundred micrometers in dimension (Fig.13 B), whereas the foam walls involved few-layer graphene sheets covered with Teflon (Fig.13 C). The foam was demonstrated to be superhydrophobic with an advanced contact angle larger than $163^{\circ}$, according to the measurement of water contact angles. Although the foam had large-sized pores, water repellency was also confirmed when the water droplets exhibited their ability to totally rebound from the surface. This superhydrophobic graphene foam exhibited potential for use in a wide variety of applications, such as self-cleaning, anti-icing, anti-fouling, and coatings for low-friction. 
Le et al. [84] prepared superhydrophobic surfaces composed of high purity, cup-stacked carbon nanotubes (CSCNTs) via a catalytic CVD approach with a contact angle of $155^{\circ}$. Transmission electron microscopy revealed that the CSCNTs' growth rate was predicted to be $20 \mu \mathrm{m} / \mathrm{min}$. CSCNTs had diameters of $80-230 \mathrm{~nm}$ containing graphene as truncated conical sheets. CSCNTs with high carbon purity above 99\% were revealed during TG analysis observations. As-grown CSCNTs were roughly aligned and slightly packed. Due to the superhydrophobic behavior, excellent flexibility, and elevated purity of such CSCNTs, they may be used to develop self-cleaning coatings and waterproof applications.

Rezaei et al. [81] produced superhydrophobic coatings with a water contact angle over $160^{\circ}$ and a sliding angle below $5^{\circ}$ by one-step CVD and modification utilizing tetraethylorthosilicate (TEOS), vinyltrimethoxysilane (VTMS) as the surface modifying molecule, and ammonium hydroxide. This is a novel method involving the simple coating of vinyl-modified silica particles on various substrates, including slides of silicone, glass, and aluminum. Vinyl groups of VTMS hindered the mechanism of silica growth. Although the film deposited from TEOS possessed a smooth surface, deposition of modified silica created a surface with high roughness. Additionally, vinyl groups of modified silica nanoparticles reduced the free energy of the surface. The surface roughness combined successfully with low surface energy to produce a superhydrophobic surface coated with a silica layer. The presence of ammonia as a catalyzer decreased the reaction temperature; this means that this method could easily be used to coat temperature-sensitive substrates.

Wu et al. [85] fabricated polyurethane (PU) @ $\mathrm{Fe}_{3} \mathrm{O}_{4} @ \mathrm{SiO}_{2} @$ Fluoropolymer (FB) sponges with water contact angles equal to $157^{\circ}$ with high magnetization and durability. The sponges were prepared using CVD of TEOS for binding the nanoparticles of $\mathrm{Fe}_{3} \mathrm{O}_{4}$ tightly onto the sponge with a subsequent step that included dip coating in a fluoropolymer (FP) aqueous solution (Fig.14 A). CVD of tetraethoxysilane produced a $\mathrm{SiO}_{2}$ thin film immobilizing the nanoparticles of $\mathrm{Fe}_{3} \mathrm{O}_{4}$ on the $\mathrm{PU}$ sponge structure. The binding of $\mathrm{Fe}_{3} \mathrm{O}_{4}$ nanoparticles, $\mathrm{SiO}_{2}$ sheet, and Fluoropolymer (FB) above the surface of the PU sponge is responsible for the superhydrophobicity (Fig.14 B-c) and high magnetization, in addition to considerable elongation. These sponges also showed excellent efficiency in oil/water separation.

\subsection{Sol-gel Technique}


A sol is typically prepared by hydrolysis of the corresponding oxide in the presence of a solvent. After network formation, a great deal of solvent will be impregnated in the network, and therefore a gel is made. There are two ways to apply a sol to the substrate: the first includes applying a gel directly on the substrate, but the other involves combining the gel with fillers, such as silica nanoparticles. Using different materials, sol-gel methods have been utilized to prepare superhydrophobic surfaces [86-90]. Zhang et al. [91] prepared superhydrophobic and very transparent silica aerogel thin film using the sol-gel technique on a glass substrate. Methanol and Methyltrimethoxysilane (MTMS) were used as raw materials to form silica aerogel on the glass substrate to fabricate superhydrophobic surfaces with contact angles up to $156.3^{\circ}$ and sliding angles of $\left(<10^{\circ}\right)$ (Fig. $15 \mathrm{~A}$ and B). Such superhydrophobic and transparent films can be used in several applications, such as cellphones, solar cells, and different pieces of electronic equipment.

Vasiljevic et al. [92] fabricated excellent superhydrophobic surfaces with antibacterial, ice-releasing, and stable thermo-oxidative properties on a cotton fiber surface using two organic and inorganic (hybrid) sol-gel precursors: 1H,1H,2H,2H-perfluorooctyltriethoxysilane (SiF) and P,Pdiphenyl-N-(3-(trimethoxysilyl)propyl) phosphinic amide (SiP) (Fig. 15 C). This work showed that the formation of a polymer network that contains a nanocomposite of two inorganicorganic (hybrid) components disseminates homogenously on a cotton fiber surface. The combination of $\mathrm{SiF}$ and $\mathrm{SiP}$ components in the two-component coating supplied the cotton fiber surface with highly effective multifunctionality.

Taurino et al. [93] prepared coatings of organic-inorganic hybrids by a multilayer method that involved multiple sol-gel processes, with the goal of obtaining superhydrophobic and highly scratch-resistant surfaces on glass. The coatings showed a water contact angle above $150^{\circ}$. Solgel processes incorporating cured vinylester resins were utilized to fabricate superhydrophobic organic-inorganic films that were highly scratch-resistant. To increase the resistance to scratching, the substrates of glass were coated with ultraviolet (UV)-curable vinylester resins and vinyltriethoxysilane (VTEOS) between the inorganic sheets, using resin as a coupling agent.

Huang and Lin [94] fabricated superhydrophobic, robust, and transparent coatings on glass substrates by using the sol-gel technique at temperature of $80^{\circ} \mathrm{C}$. The coatings were prepared in a solution of silicic acid and silica nanoparticles such that the coating roughness 
could be tuned by varying the ratio of silicic acid and silica nanoparticles. The as-deposited coating was then dipped with $1 \mathrm{H}, 1 \mathrm{H}, 2 \mathrm{H}, 2 \mathrm{H}$-perfluorooctyltrichloro silane-a low surface energy material. The team prepared superhydrophobic coatings with a water contact angle higher than $160^{\circ}$ and a sliding angle less than $10^{\circ}$ with transparent, chemical, and mechanical stability (Fig. $15 \mathrm{D})$.

Tang et al. [95] prepared fluorinated sol-gel colloid coatings by hydrolytic condensation of nanosilica sol, methyltriethoxysilane (MTES), and 3-[(Perfluorohexyl sulfonyl) amino]propyltriethoxysilane (HFTES). This combination resulted in a superhydrophobic surface with excellent contact angles of approximately $166^{\circ}$ as the content of HFTES was greater than 6 wt $\%$. The samples with HFTES content higher than $30 \mathrm{wt} \%$ exclusively showed high superhydrophobicity and anti-icing properties at freezing temperatures. This research showed that the roughness structure of a surface, in addition to high fluorinate enrichment, has considerable impact on superhydrophobic and anti-icing properties in over-cooled environments.

\subsection{Electrochemical Deposition}

Electrochemical deposition is a versatile method by which thin metallic films can be synthesized on the surface of another metal by simple electrolysis of an aqueous solution containing the desired metal ion or its complex [96]. The electrochemical deposition technique has been frequently utilized to fabricate superhydrophobic surfaces [97-100]. The preparation of superhydrophobic surfaces by this technique includes a variety of strategies that can be subdivided into two groups: 1) the process of metals oxidization or the electrodeposition of polymers and 2) processes that involve reduction, such as metal electrodeposition or galvanic deposition [99].

Khorsand et al. [101] proposed a simple electrodeposition method to prepare a superhydrophobic nickel-cobalt alloy coating on copper by a facile technique involving one- and two-step electrodeposition. Their findings demonstrated that one-step electrodeposition resulted in a Ni-Co alloy coating with superhydrophobic behavior without any modifications by low free surface energy materials. Additional elaboration of superhydrophobicity was achieved by twostep electrodeposition owing to the formation of a hierarchical and micro flower-like structure (Fig. 16 A). The freshly prepared Ni-Co coating's surface showed superhydrophilicity with a contact angle of $0^{\circ}$. The surface of the coatings prepared by one- and two-step electrodeposition 
was highly superhydrophobic with water contact angles of $158^{\circ}$ and $163^{\circ}$, respectively, after being exposed to air for two weeks. This transition was attributed to the micro/nano flower-like structure of Ni-Co films, in addition to adsorption of airborne hydrocarbon adsorbates on the films' surface. The fabricated Ni-Co coating showed self-cleaning properties, high chemical stability, and long-term durability.

Xiao et al. [102] synthesized an outstanding superhydrophobic surface containing cobalt shell-like nanostructured coatings on a copper plate. A platinum net was used as the inert anodic electrode by galvanostatic electrochemical deposition. After electroplating conditions were adjusted, cobalt (Co) coatings containing fluffy, shell-like nanocrystals proved to be highly superhydrophobic and self-cleaning (Fig. 16 B). On the other hand, the Co films with nanocrystals shaped like nanocones can hold water with high adhesion. This Co surface coating may ultimately be used as an anti-corrosive in magnetic devices.

Zhang et al. [103] used a highly successful and environmentally friendly technique to fabricate superhydrophobic surfaces by the electrodeposition of zinc followed by modification of the surface employing polypropylene (PP). The prepared superhydrophobic surface showed high superhydrophobicity with a contact angle of $170^{\circ} \pm 2^{\circ}$. The superhydrophobicity of the PPmodified zinc deposits was attributed to a lessening of the free energy of the surface due to the cooperative action of hydrophobic PP and the pored microstructure of the zinc deposits (Fig. 16 C). This study revealed that the superhydrophobic surface dramatically improved the anticorrosive effect of the zinc deposit, resulting in the possible use of such a superhydrophobic surface in anti-corrosion applications. Wang et al. [104] used an electrochemical process to fabricate superhydrophobic copper mesh coatings by electroplating the nanoparticles of copper $(\mathrm{Cu})$ onto cleaned copper mesh followed by thiol grafting. The as-prepared $\mathrm{Cu}$ mesh coating with hierarchical structures exhibited superhydrophobicity, as well as rapidly effective oil/water separation (Fig. 16 D).

\subsection{Electrospinning}

Electrospinning is a versatile technique for producing continuous fibers with diameters in the micro- and nanoscale. This technique can be extensively applied to deposit polymer films ranging from spheres to fibers. This method can be also applied to nanoparticles, metals, and 
ceramics. Additionally, it is considered an effective technique for making superhydrophobic surfaces because it enhances the surface roughness [105-110].

Using this method, Wang et al. [111] prepared superhydrophobic surfaces with high contact angles and low sliding angles, in addition to self-cleaning properties. In this study, a composite film of $\mathrm{Fe}_{3} \mathrm{O}_{4} @ \mathrm{SiO}_{2} @ \mathrm{POTS} /$ Poly (vinylidene fluoride) (PVDF) was prepared using the electrospinning method (Fig. 17 A and B). PVDF was used to make the film superparamagnetic. $\mathrm{Fe}_{3} \mathrm{O}_{4} @ \mathrm{SiO}_{2} @$ POTS nanoparticles played a large part in increasing the surface roughness. The bead-on-string PVDF was formed as the frame to support the entire film. The weight ratio of $\mathrm{Fe}_{3} \mathrm{O}_{4} @ \mathrm{SiO}_{2} @ \mathrm{POTS} / \mathrm{PVDF}$ significantly affected the composite film's morphology and structure and showed various values of superhydrophobicity and tensile strengths (Fig. $17 \mathrm{C}$ ).

Koo et al. [112] demonstrated a facile and effective technique to fabricate multifunctional and robust superhydrophobic macroporous organic/inorganic hybrid coatings and films that included phenylsilsesquiazane (PhSSQZ) electrospinning in the presence of polystyrene (PS). The freestanding PhSSQZ/PS webs, which showed hierarchical fibrous structures and exceptional chemical properties, produced a material with various practical applications as a membrane for oil/water separation, air filters with high performance, and structural coatings.

Wang et al. [113] prepared a large-scale superhydrophobic surface containing composite films with developed tensile properties via multinozzle conveyor belt electrospinning. This technique was used due to its ability to expand the area of electrospinning without limitation. By this method, three kinds of fibers were created: porous polystyrene (PS) microspheres, bead-onstring PVDF fibers, and Polyacrylonitrile PAN fibers, which were blended easily. The composite film showed high superhydrophobicity with contact angles of up to $150^{\circ}$ owing to the simultaneous presence of PS microspheres and bead-on-string PVDF fibers; thus, the surface roughness was increased. On the other hand, the presence of PAN fibers with large diameters improved the mechanical properties of the electrospun composite film considerably. This study highlighted the possibility of preparing more versatile multifunctional materials with desired properties by blending substances uniformly.

Dong et al. [114] produced a superhydrophobic surface with a contact angle of $155.5 \pm$ $1.4^{\circ}$ by using a natural material containing an amphiphilic natural protein, zein, by utilizing 
electrospinning. The superhydrophobic surface with the highest contact angle was achieved via a zein cast film (ZEF), which had been made using a solution composed of 10 wt \% of zein in $80 \%$ ethanol (Fig. 17 D and E).

\subsection{Layer-by-Layer (LBL) Deposition Technique}

Layer-by-layer (LBL) deposition is a technique in which alteration of a substrate's charge is considered the major principle; this permits the building of films with multiple layers. The LBL deposition technique is a simple and inexpensive method that makes it possible to control the thickness of each prepared layer with molecular precision. LBL generally includes layers assembled with spontaneous adsorptions of positively or negatively charged species via alternate immersion in solutions. LBL has been extensively used as a method of fabricating superhydrophobic surfaces [115-117].

Zhao et al. [118] prepared a coating with dual functions - superhydrophobicity and ultra violet (UV)-blocking properties — on a cotton fabric utilizing a hybrid layered double hydroxide (LDH) nanoplatelet intercalated with 2-hydroxy- 4-methoxybenzophenone-5-sulfonic acid (HMBS) molecules and an electrostatic LBL assembly technique (Fig. 18). The resulting HMBS@LDH hybrid had an almost neutral surface charge. The HMBS@LDH nanoplatelet was additionally modified with 3-aminopropyltriethoxy silane to enable it to carry a sufficient charge for the electrostatic LBL assembly. The nanoscale roughness that was produced by the LDH nanoplatelets in combination with low surface energy fluoroalkylsilane gave the cotton fabric surface superhydrophobicity.

Zhang et al. [119] utilized LBL self-assembly to prepare a hybrid thin film from an ionic liquid (IL) and nanoparticles of silica ( $\mathrm{SiO}_{2}$ NPs) on glass substrates, resulting in a superhydrophobic surface. The ionic liquid with a positive charge 1-dodecyl-3methylimidazoliumbromide $\left(\left[\mathrm{C}_{12} \mathrm{mim}\right] \mathrm{Br}\right)$, as well as silica nanoparticles with a negative charge, were alternatively adsorbed onto the glass substrates. The results showed that the surface roughness obtained via $\mathrm{SiO}_{2}$ NPs and the low surface energy of the ionic liquids, ( $\left.\mathrm{SiO}_{2} / \mathrm{IL}\right)_{13}$ hybrid materials, produced superhydrophobic films under the optimum size and layer numbers of silica $\left(\mathrm{SiO}_{2}\right)$, where a superhydrophobic surface with a contact angle of $152.3 \pm 5.0^{\circ}$ was obtained from $\left(\mathrm{SiO}_{2} /\left[\mathrm{C}_{12 \mathrm{mim}}\right] \mathrm{Br}\right) 13$ hybrid film. This study may open a new route to fabricate inorganic NP-IL hybrid superhydrophobic films that will preserve the IL's stability, as well as inorganic 
NPs. Potential applications include drug delivery, biomimetic materials, self-cleaning surfaces, and functional coatings.

Manabe et al. [58] produced a superhydrophobic surface with a water contact angle of $152^{\circ}$ from anti-reflective films containing chitin nanofibers (CHINFs) and silica $\mathrm{SiO}_{2}$ using the LBL self-assembly technique. The findings showed that these films offered $96.7 \%$ as a highest transmittance and 1.20 as a lowest refractive index due to the high porosity produced via the nanoparticles and nanofibers. Superhydrophobic films with $97.5 \%$ as a highest transmittance and 1.23 as a lowest reflective index, which can endow a zero reflectance, were made after modification to improve the hydrophobic rendering. This method can promote versatile materials and open a way to produce highly functionalized materials. Li et al. [120] fabricated a set of coatings that combined hierarchical nanostructures and dual roughness on stainless steel mesh and stainless steel fiber felt using the LBL self-assembly technique on silica nanoparticles of various sizes. Subsequently, the surface was exposed to chemical treatment to obtain the wetting properties required to separate oils from water. The results proved that this coated stainless steel mesh was superhydrophobic, achieving a $158^{\circ}$ contact angle; therefore, the mesh could sufficiently separate oil from water. Moreover, the stainless steel fiber felt treated with the same coatings could separate oil-in-water emulsions by the mechanism of non-sieving coalescence, which has an oil/water separation efficiency of approximately $99.4 \%$.

\subsection{Templating}

Template-dependent techniques are another imprint-related means of fabricating superhydrophobic surfaces [18,121,122]. Liu et al. [123] synthesized transparent superhydrophobic surfaces involving a film of $\mathrm{TiO}_{2}$ on a glass substrate by employing a layer of flame soot as a nanoimprint template. The $\mathrm{TiO}_{2}$ film had a structure with a roughness inverse to that of the flame soot layer, as well as high transparency features, after the nanoimprint and calcination were achieved (Fig. $19 \mathrm{~A}$ and B). 1H, 1H, 2H, 2H-per-fluorodecyltriethoxysilane (FAS) was used to modify the hydrophobic surface to produce a transparent superhydrophobic film with a high water contact angle (Fig.19 C). The prepared film exhibited high chemical stability and good mechanical resistance.

Using two different fabrication approaches, Ho et al. [124] fabricated an artificial hierarchical topography in common consumer plastics to produce superhydrophobic, self- 
cleaning plastic films. These approaches included the assembly of a nanoporous membrane in situ constructed on the micro-machined mold surface of a dome-like structure or physically placed onto a dome-shaped, macro-machined mold. The templates assembled were immediately imprinted over the thermoplastic polymers. The hierarchical topographies exhibited superhydrophobic behavior with contact angles of as much as $160^{\circ}$ and sliding angles ranging from $4^{\circ}-7^{\circ}$ for the two kinds of surfaces. The film maintained its stable superhydrophobic behavior for more than one year. An important feature of these surfaces is that hydrophobicity was achieved using common consumer plastics without adding further materials or altering the chemical nature of the surfaces.

Seo et al. [125] reported transferrable superhydrophobicity using nanorods of $\mathrm{TiO}_{2}$ on a reduced graphene oxide (rGO) film. Superhydrophobic $\mathrm{TiO}_{2}$ nanorods were first fabricated from a nanoporous template of block copolymers (BCPs). Control over the dimension and shape of BCP template nanopores was achieved by altering the molecular weights of the BCPs, which made it possible to arrange the nanostructures of $\mathrm{TiO}_{2}$ for superhydrophobicity. Since the $\mathrm{rGO}$ film endowed sufficient conveying, $\mathrm{TiO}_{2}$ nanorods were transferred onto a flexible polymer film and a metal substrate. Therefore, the designated substrate surface was effectively altered to a superhydrophobic surface without changing its inherent features.

\subsection{D printing}

Traditional manufacturing techniques, such as computerized numeric control (CNC) tools, create novel shapes of known properties of material by removing material. Conversely, additive manufacturing techniques build novel shapes by adding material [126]. For example, deposition techniques such as inkjet printing are a mature additive manufacturing process. Inkjet printing introduced the ability to move a print head controllably to a unique position in 2dimensional space. Such advancement in the deposition of a controlled dot of ink facilitated cutting-edge printing of complex shapes. Adding a third dimension to the motion of a print head created the ability to print 3D shapes from a variety of extruded materials, beginning a revolutionary additive manufacturing process called $3 \mathrm{D}$ printing.

A 3D printer has a print head that extrudes either lines or dots of material, usually a heated plastic, which hardens in place. Although 3D printers offer greater flexibility in shapes than traditional molding or injection molding processes, the technique suffers from the 
difficulties of those technologies. In particular, the plastic will change dimension as it hardens, and the material properties of the resulting part may not be consistent throughout. This can lead to an uncertain final shape and uncertain final material properties. Additive manufacturing suffers from the inverse difficulty of the removal processes, where too much material removed cannot be restored. If too much material is deposited by the $3 \mathrm{D}$ printer, the printer cannot remove the excess, resulting in dimensional inaccuracy at the top of the tolerance band.

The accuracy of print head positioning is limited by the technique employed. Traditional CNC milling processes can maintain a positional accuracy of $.02 \mathrm{~mm}$. Grinding processes typically reduce this by an order of magnitude. Higher accuracy can be achieved through gearing and through feedback of a positional measurement. The cost of such accuracy is the speed with which the print head can traverse to each position, as speed tends to vary in inverse proportion to accuracy due to the gearing. Consequently, at present, 3D printers are slow, have difficulty maintaining accuracy and repeatability of dimensions, produce variable material properties throughout a part, and have a limited number of materials available. These challenges have been overcome in traditional fabrication processes through measurement and feedback of geometry as a part is being made, through modeling of the process, and through treatment after fabrication (e.g., polishing, annealing). 3D printing will diminish its undesirable attributes over time as research and engineering improve the process.

Use of superhydrophobic materials is important in engineering, with applications in robotics [127] where 3D printing of custom robot shapes is becoming the normal means of fabrication. 3D printing has been used to create parts with superhydrophobic properties [128]. One method of achieving superhydrophobicity via 3D printing is through a repeated macro-scale shape that has such properties. Such shapes include 175 um diameter by 1300 um tall cylindrical posts [129] that support a $2 \mathrm{~mm}$ diameter water droplet (Fig. 20) and a $2.5 \mathrm{~cm}$ diameter hollow mesh ball composed of 1-1.5 mm hexagons (Fig. 21). The second method of achieving superhydrophobicity is to adapt the printer to extrude a material whose surface is superhydrophobic. For instance, one example [130] adds 2-(2-bromoisobutyryloxy)ethyl methacrylate to the resin base of a MiiCraft 3D printer. The resulting shapes are post-processed with UV curing. 
Bioprinting is a new type of 3D printing that uses the xyz stage of a conventional 3D printer. The print head is a computer controlled syringe that meters a precise droplet containing cells suspended in a liquid medium [131]. In some cases, the printer constructs a scaffold for the cells to grow [132]. This scaffold may be permanent or sacrificial. In other cases, the medium provides the structure necessary for support, such as an oil [131] or gel [133]. Although cells from superhydrophobic organisms have not yet been deployed using this technology, the technology has developed to the point where engineered organic superhydrophobic designs can be constructed.

\subsection{Direct Laser Texturing}

Direct Laser Texturing is a technique that modifies surfaces using femtosecond ( $\mathrm{fs}$ ) and nanosecond (ns) lasers. This approach is being used to create microstructures of various size and shape on different materials, thereby tailoring their properties for a wide range of applications, such as superhydrophobicity and photovoltaic cells. This technique prepares superhydrophobic surfaces on various solid substrates, including semiconductors [134], metals [135], and polymers [136]. Direct laser micro/nano texturing is a versatile and mature technique for controllable modification of surfaces of materials by a simple one-step process that does not require a clean room facility or high vacuum equipment [134-137].

Baldacchini et al. [138] submitted a simple approach for creating superhydrophobic silicon surfaces using femtosecond laser texturing. The approach consists of irradiating the silicon wafers with femtosecond laser pulses, then coating them with fluoroalkylsilane molecules. A surface morphology showed the existence of micro- and nanoscale structure due to the laser irradiation. They also demonstrated that variation in the laser fluences has a significant role in tuning the surface morphology and the wetting properties. For water, the microstructured silicon surfaces showed contact angles greater than $160^{\circ}$ and very low contact angle hysteresis. For hexadecane, the microstructuring exhibited a transition from no wetting to wetting. Relatedly, Zorba et al. [134] reported a simple method for preparing stable superhydrophobic surfaces by irradiating silicon wafers with femtosecond laser pulses, then coating them with chloroalkylsilane monolayer. This work demonstrated the possibility of achieving control over a surface's wetting properties by a systematic and reproducible variation of micro- and nanoscale roughness (Fig. 22) that mimics the lotus leaf in term of its topography and wetting behavior. Laser pulse fluence variation led to a significant altering in the wettability properties of the laser 
microstructured surfaces (Fig. 23). Such surfaces with attractive wetting characteristics can be utilized in microfluidic and self-cleaning applications.

Through cost-effective direct laser texturing, Duong et al. [139] prepared superhydrophobic surfaces on a common stainless steel (304S15) with a static contact angle of $\sim 154^{\circ}$ and a contact angle hysteresis of $\sim 4^{\circ}$. The nanosecond fiber laser employed in this work is more cost-effective and compact than the common pico-/femtosecond lasers used for this patterning; it also allows a higher processing rate. Although the processed surfaces showed hydrophilic properties immediately after fabrication, their wettability altered over time until it became non-wetting after 13 days under ambient conditions. The effect of laser power as well as scan line separation on the wettability of the textured surfaces was investigated to study optimized fabrication parameters. This study demonstrates the creation of superhydrophobic surfaces on a common type of stainless steel using nanosecond laser texturing, which can be attractive for biological applications.

\subsection{Other Significant Techniques}

Glancing Angle Deposition (GLAD) is a physical vapor deposition method in which the trajectory of the deposition flux is directed toward a rotatable substrate with an inclined angle. It has been shown that GLAD can fabricate nanostructures with special mechanical, electrical, optical, thermal, and biological applications. Recently, GLAD has emerged as an easy, inexpensive, non-hazardous, and versatile method for making perpendicular nanorods from a wide variety of materials. The nanorods usually form a porous structure on the substrate surface, and researchers have observed that they can be used to make hydrophobic surfaces from a variety of materials [140-145]. Kumar et al. [146] fabricated a superhydrophobic silver nanorod substrate via GLAD. Their study showed that the temperature of the Ag nanorod substrate plays an important part in achieving the desired contact angle. The results confirmed that the morphological difference in the silver nanorods grown at different substrate temperatures (ranging from $313 \mathrm{~K}-133 \mathrm{~K}$ ) affected the surface density and roughness of the nanostructure. The size and gap of the Ag nanorods were controlled by decorating the Ag nanorod substrate with heptadecafluoro-1-decanethiol (HDFT); this gave the surfaces high roughness and decreased the surface energy, achieving a contact angle higher than $150^{\circ}$. The highest contact angle, $164^{\circ} \pm 4$, was observed for HDFT-coated silver nanorods grown at a substrate temperature of 133K (Fig. 24). 
Anodization is a process that makes structures with huge nanopore arrays on metallic substrates with alterable shape, size, and morphology. An anodization technique has been widely used to prepare versatile nanostructures through the control of temperature, electrolytes, and an applied voltage [147-150]. Liang et al. [151] prepared a superhydrophobic Ti surface by using single-step anodization and fluorination. To make the micro/nano hierarchical structures required for a hydrophobic surface, the $\mathrm{Ti}$ substrates were anodized. Fluoroalkylsilane (FAS) modification was applied onto the Ti surface to obtain a superhydrophobic surface. This study demonstrated that proper temperature of the electrolyte and suitable applied anodization voltages were essential for balancing the dissolution and growth of the micro/nano hierarchical structure of the surface (Fig. 25 A, B, and C). The highest contact angle of the Ti substrate was $160^{\circ}$. The findings showed that the superhydrophobic Ti surface had good stability in both ambient and severe conditions, including neutral, acidic, and alkaline aqueous solutions.

In sandblasting, a stream of sand or other abrasive particles is ejected through a nozzle at a high speed to abrade the surface of a material such as metal or concrete, causing the surface to be uneven at the microscale [152]. This method has the advantages of easy preparation, low cost, and coverage of large areas of the target surface [152-154]. Fleming and Zou [155] described a simple technique to fabricate a superhydrophobic surface with long-term stability on a titanium substrate surface. Sandblasting the substrate with particles of alumina resulted in microscale roughness topography. The next step involved dip-coating by single-submersion in a colloidal silica nanoparticle solution to produce nanoscale roughness topography. The team then used chemical modifications with fluorinated carbon film with low surface energy to render the surface superhydrophobic. Due to their good stability and easy fabrication, these surfaces possess potential for use in biomedical applications.

Candle soot can be immobilized using paraffin wax, a major component in candles, in order to fabricate superhydrophobic surfaces. The method is simple yet results in durable superhydrophobic surfaces with drop impact durability, temperature and pressure stability, and $\mathrm{pH}$ tolerance [156]. A static contact angle of $\sim 162^{\circ}$ was observed for the resultant surfaces. Smoke deposition is another simple method for developing superhydrophobic surfaces; it involves depositing the silicone smoke emitted by discarded silicone combustion. The resultant surface shows excellent water repellence. A water contact angle of $164 \pm 0.8^{\circ}$ and a sliding angle 
of less than $1^{\circ}$ were measured for the surfaces. This method was employed to develop selective copper mesh material, which showed excellent water and oil separation [157].

Superhydrophobic surfaces can also be produced by a simple electroless galvanic deposition followed by surface modification with $3,3,4,4,5,5,6,6,7,7,8,8,9,9,10,10,10$ heptadecafluoro-1-decanethiol (HDFT). Copper and zinc foils can be immersed either in silver nitrate or in $\mathrm{HAuCl}_{4}$ solutions to deposit layers on the metal surfaces. The role of the surface chemistry is evident, since further modification with low energy perfluorinated thiol groups resulted in surfaces that passed McCarthy's test for $180^{\circ}$ contact angles [158].

\section{Conclusions}

Nature has inspired scientists to pursue smart solutions in several areas of technology. For example, the anti-wetting properties of the lotus leaf inspired researchers to fabricate superhydrophobic surfaces using a wide variety of techniques. This review highlights some of the progress made recently in the area of superhydrophobicity. Many fabrication methods are based on the use of surface micro-nano roughness and a material with low surface energy to obtain surfaces that mimic naturally existing superhydrophobic plants and animals. Superhydrophobic surfaces exhibit many functionally practical and valuable properties - among them, anti-icing, anti-contamination, anti-sticking, anti-fouling, and self-cleaning. The potential applications of such unique surfaces are increasingly significant in industry. Continued advances in micro/nanofabrication, new fabrication techniques, and a wide range of materials will play a large role in creating superhydrophobic surfaces for practical applications in the future.

\section{Acknowledgements}

Funding for this research was provided by the Center for Advanced Surface Engineering, under the National Science Foundation Grant No. IIA-1457888 and the Arkansas EPSCoR Program, ASSET III. The editorial assistance of Dr. Marinelle Ringer and Emity Davis is also acknowledged.

\section{References}

[1] B. Bhushan, Y.C. Jung, Natural and biomimetic artificial surfaces for superhydrophobicity, self-cleaning, low adhesion, and drag reduction, Prog. Mater. Sci. 56 
(2011) 1-108. doi:10.1016/j.pmatsci.2010.04.003.

[2] B. Bhushan, Biomimetics inspired surfaces for drag reduction and oleophobicity/philicity, Beilstein J. Nanotechnol. 2 (2011) 66-84. doi:10.3762/bjnano.2.9.

[3] M. Miwa, A. Nakajima, A. Fujishima, K. Hashimoto, T. Watanabe, Effects of the surface roughness on sliding angles of water droplets on superhydrophobic surfaces, Langmuir. 16 (2000) 5754-5760. doi:10.1021/la991660o.

[4] K. Liu, M. Cao, A. Fujishima, L. Jiang, Bio-Inspired Titanium Dioxide Materials with Special Wettability and Their Applications, Chem. Rev. (2014) --. doi:10.1021/cr4006796.

[5] X.Q. Feng, X. Gao, Z. Wu, L. Jiang, Q.S. Zheng, Superior water repellency of water strider legs with hierarchical structures: Experiments and analysis, Langmuir. 23 (2007) 4892-4896. doi:10.1021/la063039b.

[6] K. Liu, L. Jiang, Bio-inspired design of multiscale structures for function integration, Nano Today. 6 (2011) 155-175. doi:10.1016/j.nantod.2011.02.002.

[7] L. Feng, S.H. Li, Y.S. Li, H.J. Li, L.J. Zhang, J. Zhai, et al., Super-hydrophobic surfaces: From natural to artificial, Adv. Mater. 14 (2002) 1857-1860. doi:DOI 10.1002/adma.200290020.

[8] S. a. Kulinich, M. Farzaneh, Effect of contact angle hysteresis on water droplet evaporation from super-hydrophobic surfaces, Appl. Surf. Sci. 255 (2009) 4056-4060. doi:10.1016/j.apsusc.2008.10.109.

[9] S. Wang, L. Jiang, Definition of superhydrophobic states, Adv. Mater. 19 (2007) $3423-$ 3424. doi:10.1002/adma.200700934.

[10] L. Zhai, M.C. Berg, F.Ç. Cebeci, Y. Kim, J.M. Milwid, M.F. Rubner, et al., Patterned superhydrophobic surfaces: Toward a synthetic mimic of the namib desert beetle, Nano Lett. 6 (2006) 1213-1217. doi:10.1021/n1060644q.

[11] G. Wang, Z. Guo, W. Liu, Interfacial Effects of Superhydrophobic Plant Surfaces: A Review, J. Bionic Eng. 11 (2014) 325-345. doi:10.1016/S1672-6529(14)60047-0.

[12] Y.-Z. Hu, T.-B. Ma, Comprehensive Nanoscience and Technology, Elsevier, 2011. doi:10.1016/B978-0-12-374396-1.00116-1.

[13] A. Malshe, K. Rajurkar, A. Samant, H.N. Hansen, S. Bapat, W. Jiang, Bio-inspired functional surfaces for advanced applications, CIRP Ann. - Manuf. Technol. 62 (2013) 607-628. doi:10.1016/j.cirp.2013.05.008.

[14] P. Roach, N.J. Shirtcliffe, M.I. Newton, Progress in superhydrophobic surface development, Soft Matter. 4 (2008) 224. doi:10.1039/b712575p.

[15] J. Genzer, K. Efimenko, Recent developments in superhydrophobic surfaces and their relevance to marine fouling: a review., Biofouling. 22 (2006) 339-360. doi:10.1080/08927010600980223.

[16] E.I. Stratakis, V. Zorba, Biomimetic Artificial Nanostructured Surfaces, 2010. doi:10.1002/9783527610419.ntls0213. 
[17] Z. Guo, W. Liu, B.L. Su, Superhydrophobic surfaces: From natural to biomimetic to functional, J. Colloid Interface Sci. 353 (2011) 335-355. doi:10.1016/j.jcis.2010.08.047.

[18] E. Celia, T. Darmanin, E. Taffin de Givenchy, S. Amigoni, F. Guittard, Recent advances in designing superhydrophobic surfaces, J. Colloid Interface Sci. 402 (2013) 1-18. doi:10.1016/j.jcis.2013.03.041.

[19] T. Young, An Essay on the Cohesion of Fluids, Phi- Losophical Trans. R. Soc. London. 95 (1805) 65-87. http://www.jstor.org/stable/107159.

[20] R.N. Wenzel, Resistance of solid surfaces to wetting by water., J. Ind. Eng. Chem. (Washington, D. C.). 28 (1936) 988-994. doi:10.1021/ie50320a024.

[21] A.B.D. Cassie, Wettability of porous surfaces, (1944) 546-551. http://pubs.rsc.org/en/content/articlepdf/1944/tf/tf9444000546.

[22] L. Barbieri, E. Wagner, P. Hoffmann, Water wetting transition parameters of perfluorinated substrates with periodically distributed flat-top microscale obstacles, Langmuir. 23 (2007) 1723-1734. doi:10.1021/la0617964.

[23] G. Liu, L. Fu, A. V. Rode, V.S.J. Craig, Water droplet motion control on superhydrophobic surfaces: Exploiting the Wenzel-to-Cassie transition, Langmuir. 27 (2011) 2595-2600. doi:10.1021/la104669k.

[24] E. Bormashenko, R. Pogreb, G. Whyman, M. Erlich, Cassie-Wenzel wetting transition in vibrating drops deposited on rough surfaces: Is the dynamic Cassie-Wenzel wetting transition a 2D or 1D affair?, Langmuir. 23 (2007) 6501-6503. doi:10.1021/1a700935x.

[25] Y.C. Jung, B. Bhushan, Dynamic effects induced transition of droplets on biomimetic superhydrophobic surfaces, Langmuir. 25 (2009) 9208-9218. doi:10.1021/la900761u.

[26] N. a. Patankar, Transition between superhydrophobic states on rough surfaces, Langmuir. 20 (2004) 7097-7102. doi:10.1021/la049329e.

[27] A. Lafuma, D. Quéré, Superhydrophobic states., Nat. Mater. 2 (2003) 457-60. doi:10.1038/nmat924.

[28] T. Koishi, K. Yasuoka, S. Fujikawa, T. Ebisuzaki, X.C. Zeng, Coexistence and transition between Cassie and Wenzel state on pillared hydrophobic surface., Proc. Natl. Acad. Sci. U. S. A. 106 (2009) 8435-8440. doi:10.1073/pnas.0902027106.

[29] A. Marmur, Wetting on hydrophobic rough surfaces: To be heterogeneous or not to be?, Langmuir. 19 (2003) 8343-8348. doi:10.1021/la0344682.

[30] H.B. Eral, D.J.C.M. 'T Mannetje, J.M. Oh, Contact angle hysteresis: A review of fundamentals and applications, Colloid Polym. Sci. 291 (2013) 247-260. doi:10.1007/s00396-012-2796-6.

[31] H. Search, C. Journals, A. Contact, M. Iopscience, I.P. Address, Bouncing water drops, 769 (n.d.) 2-9.

[32] V. Zorba, E. Stratakis, M. Barberoglou, E. Spanakis, P. Tzanetakis, S.H. Anastasiadis, et al., Biomimetic artificial surfaces quantitatively reproduce the water repellency of a lotus leaf, Adv. Mater. 20 (2008) 4049-4054. doi:10.1002/adma.200800651. 
[33] E. Stratakis, A. Ranella, C. Fotakis, Biomimetic micro/nanostructured functional surfaces for microfluidic and tissue engineering applications, Biomicrofluidics. 5 (2011) 1-31. doi:10.1063/1.3553235.

[34] Y.C. Jung, B. Bhushan, Dynamic effects of bouncing water droplets on superhydrophobic surfaces, Langmuir. 24 (2008) 6262-6269. doi:10.1021/la8003504.

[35] C.R. Crick, I.P. Parkin, Water droplet bouncing--a definition for superhydrophobic surfaces., Chem. Commun. (Camb). 47 (2011) 12059-61. doi:10.1039/c1cc14749h.

[36] Y. Lee, S.H. Park, K.B. Kim, J.K. Lee, Fabrication of hierarchical structures on a polymer surface to mimic natural superhydrophobic surfaces, Adv. Mater. 19 (2007) 2330-2335. doi:10.1002/adma.200700820.

[37] M. Nosonovsky, B. Bhushan, Biomimetic superhydrophobic surfaces: Multiscale approach, Nano Lett. 7 (2007) 2633-2637. doi:10.1021/n1071023f.

[38] W.B. Y.Y. Yana, N. Gaoa, Mimicking natural superhydrophobic surfaces and grasping the wetting process: A review on recent progress in preparing superhydrophobic surfaces, Adv. Colloid Interface Sci. 169 (2011) 80-105. doi:10.1016/j.cis.2011.08.005.

[39] T. Darmanin, F. Guittard, Superhydrophobic and superoleophobic properties in nature, Mater. Today. 00 (2015) 1-13. doi:10.1016/j.mattod.2015.01.001.

[40] W. Barthlott, C. Neinhuis, Purity of the sacred lotus, or escape from contamination in biological surfaces, Planta. 202 (1997) 1-8. doi:10.1007/s004250050096.

[41] B. Bhushan, Y.C. Jung, K. Koch, Micro-, nano- and hierarchical structures for superhydrophobicity, self-cleaning and low adhesion, Philos. Trans. R. Soc. A Math. Phys. Eng. Sci. 367 (2009) 1631-1672. doi:10.1098/rsta.2009.0014.

[42] J.B.K. Law, A.M.H. Ng, A.Y. He, H.Y. Low, Bioinspired ultrahigh water pinning nanostructures, Langmuir. 30 (2014) 325-331. doi:10.1021/la4034996.

[43] Z. Guo, W. Liu, Biomimic from the superhydrophobic plant leaves in nature: Binary structure and unitary structure, Plant Sci. 172 (2007) 1103-1112. doi:10.1016/j.plantsci.2007.03.005.

[44] W. Barthlott, T. Schimmel, S. Wiersch, K. Koch, M. Brede, M. Barczewski, et al., The salvmia paradox: superhydrophobic surfaces with hydrophilic pins for air retention under water, Adv. Mater. 22 (2010) 2325-2328. doi:10.1002/adma.200904411.

[45] L. Zheng, Y., Gao, X., Jiang, Directional adhesion of superhydrophobic butterfly wings, Soft Matter. 3 (2007) 178-182.

[46] Y. Zheng, X. Gao, L. Jiang, Directional adhesion of superhydrophobic butterfly wings, Soft Matter. 3 (2007) 178. doi:10.1039/b612667g.

[47] a R. Parker, C.R. Lawrence, Water capture by a desert beetle., Nature. 414 (2001) 33-34. doi:10.1038/35102108.

[48] D.W. Bechert, M. Bruse, W. Hage, Experiments with three-dimensional riblets as an idealized model of shark skin, Exp. Fluids. 28 (2000) 403-412.

doi: $10.1007 / \mathrm{s} 003480050400$. 
[49] P. Ball, Engineering shark skin and other solutions, Nature. 400 (1999) 507-+. doi:10.1038/22883.

[50] B. Dean, B. Bhushan, Shark-skin surfaces for fluid-drag reduction in turbulent flow: a review., Philos. Trans. A. Math. Phys. Eng. Sci. 368 (2010) 4775-4806.

doi:10.1098/rsta.2010.0294.

[51] J. Genzer, K. Efimenko, Recent developments in superhydrophobic surfaces and their relevance to marine fouling: a review, Biofouling. 22 (2006) 339-360. doi:10.1080/08927010600980223.

[52] W.R. Hansen, K. Autumn, Evidence for self-cleaning in gecko setae., Proc. Natl. Acad. Sci. U. S. A. 102 (2005) 385-389. doi:10.1073/pnas.0408304102.

[53] K. Autumn, Y. a Liang, S.T. Hsieh, W. Zesch, W.P. Chan, T.W. Kenny, et al., Adhesive force of a single gecko foot-hair., Nature. 405 (2000) 681-685. doi:10.1038/35015073.

[54] X.-M. Li, D. Reinhoudt, M. Crego-Calama, What do we need for a superhydrophobic surface? A review on the recent progress in the preparation of superhydrophobic surfaces., Chem. Soc. Rev. 36 (2007) 1350-1368. doi:10.1039/b602486f.

[55] W.L. Min, B. Jiang, P. Jiang, Bioinspired self-cleaning antireflection coatings, Adv. Mater. 20 (2008) 3914-3918. doi:10.1002/adma.200800791.

[56] T. Sun, L. Feng, X. Gao, L. Jiang, Bioinspired surfaces with special wettability, Acc. Chem. Res. 38 (2005) 644-652. doi:10.1021/ar040224c.

[57] X. Gao, X. Yan, X. Yao, L. Xu, K. Zhang, J. Zhang, et al., The dry-style antifogging properties of mosquito compound eyes and artificial analogues prepared by soft lithography, Adv. Mater. 19 (2007) 2213-2217. doi:10.1002/adma.200601946.

[58] K. Manabe, S. Nishizawa, K. Kyung, S. Shiratori, Optical Phenomena and Anti-Frosting Property on Biomimetics Slippery Fluid-Infused Antireflective Films via Layer-by-Layer by Comparing with Superhydrophobic and Antireflective Films., ACS Appl. Mater. Interfaces. (2014). doi:10.1021/am503352x.

[59] F. Xia, L. Jiang, Bio-inspired, smart, multiscale interfacial materials, Adv. Mater. 20 (2008) 2842-2858. doi:10.1002/adma.200800836.

[60] B. Ziaie, A. Baldi, M. Lei, Y. Gu, R. a. Siegel, Hard and soft micromachining for BioMEMS: Review of techniques and examples of applications in microfluidics and drug delivery, Adv. Drug Deliv. Rev. 56 (2004) 145-172. doi:10.1016/j.addr.2003.09.001.

[61] R.F. Pease, S.Y. Chou, Lithography and other patterning techniques for future electronics, Proc. IEEE. 96 (2008) 248-270. doi:10.1109/JPROC.2007.911853.

[62] N. Kawasegi, N. Morita, S. Yamada, N. Takano, T. Oyama, S. Momota, et al., Depth control of a silicon structure fabricated by $100 \mathrm{qkeV}$ Ar ion beam lithography, Appl. Surf. Sci. 253 (2007) 3284-3291. doi:10.1016/j.apsusc.2006.07.037.

[63] A. Milionis, I. Bayer, D. Fragouli, Combination of Lithography and Coating Methods for Surface Wetting Control, (2013). http://cdn.intechopen.com/pdfs/45206/InTechCombination_of_lithography_and_coating_methods_for_surface_wetting_control.pdf 
(accessed January 19, 2016).

[64] Y. Lai, C. Lin, H. Wang, J. Huang, H. Zhuang, L. Sun, Superhydrophilicsuperhydrophobic micropattern on $\mathrm{TiO} 2$ nanotube films by photocatalytic lithography, Electrochem. Commun. 10 (2008) 387-391. doi:10.1016/j.elecom.2007.12.020.

[65] P. Hanarp, D.S. Sutherland, J. Gold, B. Kasemo, Control of nanoparticle film structure for colloidal lithography, Colloids Surfaces A Physicochem. Eng. Asp. 214 (2003) 23-36. doi:10.1016/S0927-7757(02)00367-9.

[66] G.A. DeRose, L. Zhu, J.K.S. Poon, A. Yariv, A. Scherer, Periodic sub-wavelength electron beam lithography defined photonic crystals for mode control in semiconductor lasers, Microelectron. Eng. 85 (2008) 758-760. doi:10.1016/j.mee.2007.12.030.

[67] W. Li, Z. Wang, D. Wang, Z. Zhang, L. Zhao, D. Li, et al., Superhydrophobic dual microand nanostructures fabricated by direct laser interference lithography, Opt. Eng. 53 (2014) 034109. doi:10.1117/1.OE.53.3.034109.

[68] S.M. Kang, S. Hwang, S.H. Jin, C.H. Choi, J. Kim, B.J. Park, et al., A rapid one-step fabrication of patternable superhydrophobic surfaces driven by marangoni instability, Langmuir. 30 (2014) 2828-2834. doi:10.1021/la500266f.

[69] H.J. Choi, S. Choo, J.H. Shin, K.I. Kim, H. Lee, Fabrication of superhydrophobic and oleophobic surfaces with overhang structure by reverse nanoimprint lithography, J. Phys. Chem. C. 117 (2013) 24354-24359. doi:10.1021/jp4070399.

[70] K.P. Adhi, R.L. Owings, T. a. Railkar, W.D. Brown, a. P. Malshe, Femtosecond ultraviolet $(248 \mathrm{~nm}$ ) excimer laser processing of Teflon (PTFE), Appl. Surf. Sci. 218 (2003) 17-23. doi:10.1016/S0169-4332(03)00586-5.

[71] Y. Xiu, S. Zhang, V. Yelundur, A. Rohatgi, D.W. Hess, C.P. Wong, Superhydrophobic and low light reflectivity silicon surfaces fabricated by hierarchical etching, Langmuir. 24 (2008) 10421-10426. doi:10.1021/la801206m.

[72] Z. Wang, Q. Li, Z. She, F. Chen, L. Li, X. Zhang, et al., Facile and fast fabrication of superhydrophobic surface on magnesium alloy, Appl. Surf. Sci. 271 (2013) 182-192. doi:10.1016/j.apsusc.2013.01.158.

[73] M. Ruan, W. Li, B. Wang, B. Deng, F. Ma, Z. Yu, Preparation and anti-icing behavior of superhydrophobic surfaces on aluminum alloy substrates, Langmuir. 29 (2013) 84828491. doi:10.1021/la400979d.

[74] Z. Guo, F. Zhou, J. Hao, W. Liu, Effects of system parameters on making aluminum alloy lotus, J. Colloid Interface Sci. 303 (2006) 298-305. doi:10.1016/j.jcis.2006.06.067.

[75] R. Liao, Z. Zuo, C. Guo, Y. Yuan, A. Zhuang, Fabrication of superhydrophobic surface on aluminum by continuous chemical etching and its anti-icing property, Appl. Surf. Sci. 317 (2014) 701-709. doi:10.1016/j.apsusc.2014.08.187.

[76] J. Song, W. Xu, X. Liu, Y. Lu, Z. Wei, L. Wu, Ultrafast fabrication of rough structures required by superhydrophobic surfaces on Al substrates using an immersion method, 
Chem. Eng. J. 211-212 (2012) 143-152. doi:10.1016/j.cej.2012.09.094.

[77] K. Li, X. Zeng, H. Li, X. Lai, A study on the fabrication of superhydrophobic iron surfaces by chemical etching and galvanic replacement methods and their anti-icing properties, Appl. Surf. Sci. 346 (2015) 458-463. doi:10.1016/j.apsusc.2015.03.130.

[78] H.C. Barshilia, N. Gupta, Superhydrophobic polytetrafluoroethylene surfaces with leaflike micro-protrusions through Ar + O2 plasma etching process, Vacuum. 99 (2014) 42 48. doi:10.1016/j.vacuum.2013.04.020.

[79] K. Reinhold-López, A. Braeuer, B. Romann, N. Popovska-Leipertz, A. Leipertz, In Situ Raman Monitoring of the Formation and Growth of Carbon Nanotubes via Chemical Vapor Deposition, Procedia Eng. 102 (2015) 190-200. doi:10.1016/j.proeng.2015.01.126.

[80] Z. Zheng, Z. Gu, R. Huo, Y. Ye, Superhydrophobicity of polyvinylidene fluoride membrane fabricated by chemical vapor deposition from solution, Appl. Surf. Sci. 255 (2009) 7263-7267. doi:10.1016/j.apsusc.2009.03.084.

[81] S. Rezaei, I. Manoucheri, R. Moradian, B. Pourabbas, One-step chemical vapor deposition and modification of silica nanoparticles at the lowest possible temperature and superhydrophobic surface fabrication, Chem. Eng. J. 252 (2014) 11-16. doi:10.1016/j.cej.2014.04.100.

[82] L.-Y. Meng, S.-J. Park, Effect of growth of graphite nanofibers on superhydrophobic and electrochemical properties of carbon fibers, Mater. Chem. Phys. 132 (2012) 324-329. doi:10.1016/j.matchemphys.2011.11.024.

[83] E. Singh, Z. Chen, F. Houshmand, W. Ren, Y. Peles, H.M. Cheng, et al., Superhydrophobic graphene foams, Small. 9 (2013) 75-80. doi:10.1002/smll.201201176.

[84] S.S. Li, P.X. Hou, C. Liu, Growth of a cup-stacked carbon nanotube carpet with a superhydrophobic surface, Xinxing Tan Cailiao/New Carbon Mater. 28 (2013) 295-299. doi:10.1016/S1872-5805(13)60083-9.

[85] L. Wu, L. Li, B. Li, J. Zhang, A. Wang, Magnetic, Durable, and Superhydrophobic

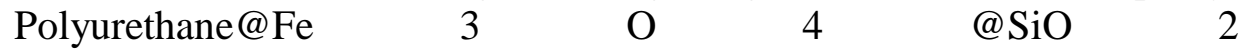
@Fluoropolymer Sponges for Selective Oil Absorption and Oil/Water Separation, ACS Appl. Mater. Interfaces. (2015) 150223100257003. doi:10.1021/am5091353.

[86] M. Hikita, K. Tanaka, T. Nakamura, T. Kajiyama, A. Takahara, Super-liquid-repellent surfaces prepared by colloidal silica nanoparticles covered with fluoroalkyl groups, Langmuir. 21 (2005) 7299-7302. doi:10.1021/la050901r.

[87] A. Qu, X. Wen, P. Pi, J. Cheng, Z. Yang, Morphologies and Superhydrophobicity of Hybrid Film Surfaces Based on Silica and Fluoropolymer, J. Mater. Sci. Technol. 24 (2008) 693-699. http://d.wanfangdata.com.cn/Periodical_clkxjsxb-e200805003.aspx.

[88] S.C.G. A. Venkateswara Raoa, Annaso B. Gurava, Sanjay S. Latthea, Rajiv S. Vhatkara, Hiroaki Imaib, Charles Kappensteinc, P.B. Waghd, Water repellent porous silica films by sol-gel dip coating method, J. Colloid Interface Sci. 352 (2010) 30-35.

doi:10.1016/j.jcis.2010.08.003. 
[89] S.S. Latthe, D.Y. Nadargi, A. Venkateswara Rao, TMOS based water repellent silica thin films by co-precursor method using TMES as a hydrophobic agent, Appl. Surf. Sci. 255 (2009) 3600-3604. doi:10.1016/j.apsusc.2008.10.005.

[90] A.V. Rao, A.B. Gurav, S.S. Latthe, R.S. Vhatkar, H. Imai, C. Kappenstein, et al., Water repellent porous silica films by sol-gel dip coating method., J. Colloid Interface Sci. 352 (2010) 30-5. doi:10.1016/j.jcis.2010.08.003.

[91] T.T. Zhang, J. Huang, R.Z. Zhang, P.Y. Mao, P. Fang, Highly Transparent Superhydrophobic Surfaces from Silica Aerogel by Sol-Gel Method, Adv. Mater. Res. 756-759 (2013) 150-153. doi:10.4028/www.scientific.net/AMR.756-759.150.

[92] J. Vasiljević, B. Tomšič, I. Jerman, B. Orel, G. Jakša, J. Kovač, et al., Multifunctional superhydrophobic/oleophobic and flame-retardant cellulose fibres with improved icereleasing properties and passive antibacterial activity prepared via the sol-gel method, J. Sol-Gel Sci. Technol. 70 (2014) 385-399. doi:10.1007/s10971-014-3294-8.

[93] R. Taurino, E. Fabbri, D. Pospiech, A. Synytska, M. Messori, Preparation of scratch resistant superhydrophobic hybrid coatings by sol-gel process, Prog. Org. Coatings. (2014) 3-9. doi:10.1016/j.porgcoat.2014.05.009.

[94] W.-H. Huang, C.-S. Lin, Robust superhydrophobic transparent coatings fabricated by a low-temperature sol-gel process, Appl. Surf. Sci. 305 (2014) 702-709. doi:10.1016/j.apsusc.2014.03.179.

[95] Y. Tang, Q. Zhang, X. Zhan, F. Chen, Superhydrophobic and anti-icing properties at overcooled temperature of a fluorinated hybrid surface prepared via a sol-gel process, Soft Matter. (2015). doi:10.1039/C5SM00674K.

[96] C.R.K. Rao, D.C. Trivedi, Chemical and electrochemical depositions of platinum group metals and their applications, Coord. Chem. Rev. 249 (2005) 613-631. doi:10.1016/j.ccr.2004.08.015.

[97] M. Ma, R.M. Hill, Superhydrophobic surfaces, Curr. Opin. Colloid Interface Sci. 11 (2006) 193-202. doi:10.1016/j.cocis.2006.06.002.

[98] S. Khorsand, K. Raeissi, F. Ashrafizadeh, Corrosion resistance and long-term durability of super-hydrophobic nickel film prepared by electrodeposition process, Appl. Surf. Sci. 305 (2014) 498-505. doi:10.1016/j.apsusc.2014.03.123.

[99] T. Darmanin, E.T. De Givenchy, S. Amigoni, F. Guittard, Superhydrophobic surfaces by electrochemical processes, Adv. Mater. 25 (2013) 1378-1394. doi:10.1002/adma.201204300.

[100] X. Zhang, F. Shi, X. Yu, H. Liu, Y. Fu, Z. Wang, et al., Polyelectrolyte Multilayer as Matrix for Electrochemical Deposition of Gold Clusters: Toward Super-Hydrophobic Surface, J. Am. Chem. Soc. 126 (2004) 3064-3065. doi:10.1021/ja0398722.

[101] S. Khorsand, K. Raeissi, F. Ashrafizadeh, M. a. Arenas, Super-hydrophobic nickel-cobalt alloy coating with micro-nano flower-like structure, Chem. Eng. J. 273 (2015) 638-646. doi:10.1016/j.cej.2015.03.076. 
[102] H. Xiao, A. Hu, T. Hang, M. Li, Electrodeposited nanostructured cobalt film and its dual modulation of both superhydrophobic property and adhesiveness, Appl. Surf. Sci. 324 (2015) 319-323. doi:10.1016/j.apsusc.2014.10.156.

[103] X. Zhang, J. Liang, B. Liu, Z. Peng, Preparation of superhydrophobic zinc coating for corrosion protection, Colloids Surfaces A Physicochem. Eng. Asp. 454 (2014) 113-118. doi:10.1016/j.colsurfa.2014.04.029.

[104] B. Wang, Z. Guo, Superhydrophobic copper mesh films with rapid oil/water separation properties by electrochemical deposition inspired from butterfly wing, Appl. Phys. Lett. 103 (2013). doi:10.1063/1.4817922.

[105] J. Lin, Y. Cai, X. Wang, B. Ding, J. Yu, M. Wang, Fabrication of biomimetic superhydrophobic surfaces inspired by lotus leaf and silver ragwort leaf., Nanoscale. 3 (2011) 1258-1262. doi:10.1039/c0nr00812e.

[106] X. Wang, F. Cui, J. Lin, B. Ding, J. Yu, S.S. Al-Deyab, Functionalized nanoporous TiO 2 fibers on quartz crystal microbalance platform for formaldehyde sensor, Sensors Actuators, B Chem. 171-172 (2012) 658-665. doi:10.1016/j.snb.2012.05.050.

[107] S.H. Park, S.M. Lee, H.S. Lim, J.T. Han, D.R. Lee, H.S. Shin, et al., Robust superhydrophobic mats based on electrospun crystalline nanofibers combined with a silane precursor, ACS Appl. Mater. Interfaces. 2 (2010) 658-662. doi:10.1021/am100005x.

[108] M. Ma, Y. Mao, M. Gupta, K.K. Gleason, G.C. Rutledge, Superhydrophobic fabrics produced by electrospinning and chemical vapor deposition, Macromolecules. 38 (2005) 9742-9748. doi:10.1021/ma0511189.

[109] H.S. Lim, J.H. Baek, K. Park, H.S. Shin, J. Kim, J.H. Cho, Multifunctional hybrid fabrics with thermally stable superhydrophobicity, Adv. Mater. 22 (2010) 2138-2141. doi:10.1002/adma.200903074.

[110] M. Ma, R.M. Hill, J.L. Lowery, S. V. Fridrikh, G.C. Rutledge, Electrospun poly(styreneblock-dimethylsiloxane) block copolymer fibers exhibiting superhydrophobicity, Langmuir. 21 (2005) 5549-5554. doi:10.1021/la047064y.

[111] S. Wang, Q. Liu, Y. Zhang, S. Wang, Y. Li, Q. Yang, et al., Preparation of a multifunctional material with superhydrophobicity, superparamagnetism, mechanical stability and acids-bases resistance by electrospinning, Appl. Surf. Sci. 279 (2013) 150158. doi:10.1016/j.apsusc.2013.04.060.

[112] S.H. Koo, S.G. Lee, H. Bong, Y.J. Kwark, K. Cho, H.S. Lim, et al., Robust multifunctional superhydrophobic organic-inorganic hybrid macroporous coatings and films, Polym. (United Kingdom). 55 (2014) 2661-2666. doi:10.1016/j.polymer.2014.03.046.

[113] S. Wang, Y. Yang, Y. Zhang, X. Fei, C. Zhou, Y. Zhang, et al., Fabrication of large-scale superhydrophobic composite films with enhanced tensile properties by multinozzle conveyor belt electrospinning, J. Appl. Polym. Sci. 131 (2014) n/a-n/a. 
doi:10.1002/app.39735.

[114] F. Dong, M. Zhang, W.-W. Tang, Y. Wang, Formation and Mechanism of Superhydrophobic/Hydrophobic Surfaces Made from Amphiphiles through DropletMediated Evaporation-Induced Self-Assembly, J. Phys. Chem. B. (2015) 150409083834009. doi:10.1021/acs.jpcb.5b00011.

[115] H. Benten, N. Kudo, H. Ohkita, S. Ito, Layer-by-layer deposition films of copper phthalocyanine derivative; their photoelectrochemical properties and application to solution-processed thin-film organic solar cells, Thin Solid Films. 517 (2009) 2016-2022. doi:10.1016/j.tsf.2008.09.102.

[116] J. Hong, S.W. Kang, Carbon decorative coatings by dip-, spin-, and spray-assisted layerby-layer assembly deposition., J. Nanosci. Nanotechnol. 11 (2011) 7771-6. http://www.ncbi.nlm.nih.gov/pubmed/22097485 (accessed January 19, 2016).

[117] M.M. El-Nahass, a. M. Farag, K.F. Abd El-Rahman, a. a a Darwish, Dispersion studies and electronic transitions in nickel phthalocyanine thin films, Opt. Laser Technol. 37 (2005) 513-523. doi:10.1016/j.optlastec.2004.08.016.

[118] Y. Zhao, Z. Xu, X. Wang, T. Lin, Superhydrophobic and UV-blocking cotton fabrics prepared by layer-by-layer assembly of organic UV absorber intercalated layered double hydroxides, Appl. Surf. Sci. 286 (2013) 364-370. doi:10.1016/j.apsusc.2013.09.092.

[119] C. Zhang, S. Zhang, P. Gao, H. Ma, Q. Wei, Superhydrophobic hybrid films prepared from silica nanoparticles and ionic liquids via layer-by-layer self-assembly, Thin Solid Films. 570 (2014) 27-32. doi:10.1016/j.tsf.2014.08.045.

[120] X. Li, D. Hu, K. Huang, C. Yang, Hierarchical rough surfaces formed by LBL selfassembly for oil-water separation, J. Mater. Chem. A. 2 (2014) 11830. doi:10.1039/C4TA01569J.

[121] W. Ye, Q. Shi, J. Hou, J. Jin, Q. Fan, S.-C. Wong, et al., Superhydrophobic coating of elastomer on different substrates using a liquid template to construct a biocompatible and antibacterial surface, J. Mater. Chem. B. 2 (2014) 7186-7191. doi:10.1039/C4TB01126K.

[122] M.E. Abdelsalam, P.N. Bartlett, T. Kelf, J. Baumberg, Wetting of regularly structured gold surfaces, Langmuir. 21 (2005) 1753-1757. doi:10.1021/la047468q.

[123] S. Liu, M. Sakai, B. Liu, C. Terashima, K. Nakata, A. Fujishima, Facile synthesis of transparent superhydrophobic titania coating by using soot as a nanoimprint template, RSC Adv. 3 (2013) 22825. doi:10.1039/c3ra43798a.

[124] A.Y.Y. Ho, E. Luong Van, C.T. Lim, S. Natarajan, N. Elmouelhi, H.Y. Low, et al., Lotus bioinspired superhydrophobic, self-cleaning surfaces from hierarchically assembled templates, J. Polym. Sci. Part B Polym. Phys. 52 (2014) 603-609. doi:10.1002/polb.23461.

[125] M.-S. Seo, J.-H. Kim, S.-S. Kim, H. Kang, B.-H. Sohn, Transferrable superhydrophobic TiO 2 nanorods on reduced graphene oxide films using block copolymer templates, Nanotechnology. 26 (2015) 165302. doi:10.1088/0957-4484/26/16/165302.

[126] T. Snyder, M. Andrews, 3D Systems' Technology Overview and New Applications in 
Manufacturing, Engineering, Science, and Education, 3D Print. .... 1 (2014) 169-176. doi:10.1089/3dp.2014.1502.

[127] J. Yan, K. Yang, T. Wang, J. Zhao, Research on Design and Jumping Performance of a New Water-jumping Robot Imitating Water Striders *, (2015) 353-358.

[128] A.M. Lyons, B.L. Alcatel-lucent, B.L. Alcatel-lucent, M. Hill, Three-dimensional superhydrophobic structures printed using solid freeform fabrication tools John Mullins Mark Barahman and Itay Erlich Todd Salamon, 3 (2013) 89-104.

[129] A.M. Lyons, J. Mullins, M. Barahman, I. Erlich, T. Salamon, Three-dimensional superhydrophobic structures printed using solid freeform fabrication tools, Int. J. Rapid Manuf. (2013).

http://www.inderscienceonline.com/doi/abs/10.1504/IJRAPIDM.2013.053683 (accessed January 24, 2016).

[130] X. Wang, X. Cai, Q. Guo, T. Zhang, B. Kobe, J. Yang, i3DP, a robust 3D printing approach enabling genetic post-printing surface modification., Chem. Commun. (Camb). 49 (2013) 10064-6. doi:10.1039/c3cc45817b.

[131] N. Mobilization, S. America, E.O. Wilson, R.W. Wrangham, S. Bowles, M.W. Feldman, et al., Special section, 336 (2012) 879-885.

[132] G. Villar, A.D. Graham, H. Bayley, A tissue-like printed material., Science. 340 (2013) 48-52. doi:10.1126/science.1229495.

[133] N.E. Fedorovich, I. Swennen, J. Girones, L. Moroni, C.A. Van Blitterswijk, E. Schacht, et al., Evaluation of photocrosslinked lutrol hydrogel for tissue printing applications, Biomacromolecules. 10 (2009) 1689-1696. doi:10.1021/bm801463q.

[134] V. Zorba, E. Stratakis, M. Barberoglou, E. Spanakis, P. Tzanetakis, C. Fotakis, Tailoring the wetting response of silicon surfaces via fs laser structuring, Appl. Phys. A Mater. Sci. Process. 93 (2008) 819-825. doi:10.1007/s00339-008-4757-y.

[135] A.M. Kietzig, S.G. Hatzikiriakos, P. Englezos, Patterned superhydrophobic metallic surfaces, Langmuir. 25 (2009) 4821-4827. doi:10.1021/la8037582.

[136] H. Pazokian, a Selimis, J. Barzin, S. Jelvani, M. Mollabashi, C. Fotakis, et al., Tailoring the wetting properties of polymers from highly hydrophilic to superhydrophobic using UV laser pulses, J. Micromechanics Microengineering. 22 (2012) 035001. doi:10.1088/09601317/22/3/035001.

[137] V. Zorba, L. Persano, D. Pisignano, A. Athanassiou, E. Stratakis, R. Cingolani, et al., Making silicon hydrophobic: wettability control by two-lengthscale simultaneous patterning with femtosecond laser irradiation, Nanotechnology. 17 (2006) 3234-3238. doi:10.1088/0957-4484/17/13/026.

[138] T. Baldacchini, J.E. Carey, M. Zhou, E. Mazur, Superhydrophobic surfaces prepared by microstructuring of silicon using a femtosecond laser., Langmuir. 22 (2006) 4917-4919. doi:10.1021/la053374k. 
[139] V. Duong, J. Thomas, W. Robert, J. Patrick, D. Jonathan, R.W. Kay, et al., Laser textured superhydrophobic surfaces and their applications for homogeneous spot deposition, 365 (2016) 153-159. doi:10.1016/j.apsusc.2016.01.019.

[140] S. V. Kesapragada, D. Gall, Anisotropic broadening of Cu nanorods during glancing angle deposition, Appl. Phys. Lett. 89 (2006) 1-3. doi:10.1063/1.2388861.

[141] Y.P. Zhao, D.X. Ye, G.C. Wang, T.M. Lu, Designing nanostructures by glancing angle deposition, Proc. SPIE Vol. 5219 Nanotub. Nanowires. 5219 (2003) 59-73. doi: $10.1117 / 12.505253$.

[142] H.J. Gwon, Y. Park, C.W. Moon, S. Nahm, S.J. Yoon, S.Y. Kim, et al., Superhydrophobic and antireflective nanograss-coated glass for high performance solar cells, Nano Res. 7 (2014) 670-678. doi:10.1007/s12274-014-0427-x.

[143] D.P. Singh, J.P. Singh, Controlled growth of standing Ag nanorod arrays on bare Si substrate using glancing angle deposition for self-cleaning applications, Appl. Phys. A Mater. Sci. Process. 114 (2014) 1189-1193. doi:10.1007/s00339-013-7819-8.

[144] G.K. Kannarpady, K.R. Khedir, H. Ishihara, J. Woo, O.D. Oshin, S. Trigwell, et al., Controlled growth of self-organized hexagonal arrays of metallic nanorods using template-assisted glancing angle deposition for superhydrophobic applications, ACS Appl. Mater. Interfaces. 3 (2011) 2332-2340. doi:10.1021/am200251n.

[145] X. Zhou, D. Xu, G. Yang, Q. Zhang, J. Shen, J. Lu, et al., Highly exothermic and superhydrophobic Mg/fluorocarbon core/shell nanoenergetic arrays, ACS Appl. Mater. Interfaces. 6 (2014) 10497-10505. doi:10.1021/am502078e.

[146] S. Kumar, P. Goel, D.P. Singh, J.P. Singh, Fabrication of superhydrophobic silver nanorods array substrate using glancing angle deposition, AIP Conf. Proc. 1591 (2014) 872-874. doi:10.1063/1.4872786.

[147] a. Bayat, M. Ebrahimi, a. Nourmohammadi, a. Z. Moshfegh, Wettability properties of PTFE/ZnO nanorods thin film exhibiting UV-resilient superhydrophobicity, Appl. Surf. Sci. 341 (2015) 92-99. doi:10.1016/j.apsusc.2015.02.197.

[148] Y. Gao, Y. Sun, D. Guo, Facile fabrication of superhydrophobic surfaces with low roughness on Ti-6Al-4V substrates via anodization, Appl. Surf. Sci. 314 (2014) 754-759. doi:10.1016/j.apsusc.2014.07.059.

[149] M. Song, Y. Liu, S. Cui, L. Liu, M. Yang, Fabrication and icing property of superhydrophilic and superhydrophobic aluminum surfaces derived from anodizing aluminum foil in a sodium chloride aqueous solution, Appl. Surf. Sci. 283 (2013) 19-24. doi:10.1016/j.apsusc.2013.05.088.

[150] B.Y. Jeong, E.H. Jung, J.H. Kim, Fabrication of superhydrophobic niobium pentoxide thin films by anodization, Appl. Surf. Sci. 307 (2014) 28-32. doi:10.1016/j.apsusc.2014.03.111.

[151] J. Liang, K. Liu, D. Wang, H. Li, P. Li, S. Li, et al., Facile fabrication of superhydrophilic/superhydrophobic surface on titanium substrate by single-step anodization and fluorination, Appl. Surf. Sci. 338 (2015) 126-136. 
doi:10.1016/j.apsusc.2015.02.117.

[152] D. Kim, J. Kim, H.C. Park, K.-H. Lee, W. Hwang, A superhydrophobic dual-scale engineered lotus leaf, J. Micromechanics Microengineering. 18 (2007) 015019. doi:10.1088/0960-1317/18/1/015019.

[153] M. Psarski, G. Celichowski, J. Marczak, K. Gumowski, G.B. Sobieraj, Superhydrophobic dual-sized filler epoxy composite coatings, Surf. Coatings Technol. 225 (2013) 66-74. doi:10.1016/j.surfcoat.2013.03.017.

[154] M. Alonso Frank, A.R. Boccaccini, S. Virtanen, A facile and scalable method to produce superhydrophic stainless steel surface, Appl. Surf. Sci. 311 (2014) 753-757. doi:10.1016/j.apsusc.2014.05.152.

[155] R. a. Fleming, M. Zou, Silica nanoparticle-based films on titanium substrates with longterm superhydrophilic and superhydrophobic stability, Appl. Surf. Sci. 280 (2013) 820827. doi:10.1016/j.apsusc.2013.05.068.

[156] K. Seo, M. Kim, D.H. Kim, Candle-based process for creating a stable superhydrophobic surface, Carbon N. Y. 68 (2014) 583-596. doi:10.1016/j.carbon.2013.11.038.

[157] W. Qiu, D. Xu, B. Liu, L. Shen, Q. Guo, Fabrication of superhydrophobic surfaces by smoke deposition and application in oil-water separation, RSC Adv. 5 (2015) 71329 71335. doi:10.1039/C5RA12287B.

[158] I.A. Larmour, S.E.J. Bell, G.C. Saunders, Remarkably Simple Fabrication of Superhydrophobic Surfaces Using Electroless Galvanic Deposition, Angew. Chemie Int. Ed. 46 (2007) 1710-1712. doi:10.1002/anie.200604596.

\section{Fig. captions}

Fig. 1. A schematic diagram exhibits the forces at the three phases of liquid/vapor/solid contact line (CL) of a droplet of liquid resting on a solid surface.

Fig. 2. A diagram shows the Wenzel`s model.

Fig. 3. A diagram shows the heterogonous wetting behavior described by Cassie and Baxter. 
Fig. 4. The diagram shows the transition from Wenzel to Cassie state and vice versa, which occurs due to external effects.

Fig. 5. SEM images of superhydrophobic surfaces in nature: (A) Lotus leaf [41]. Copyright (2009), The Royal Society. (B) Taro leaf [43]. Copyright (2007), Elsevier. (C) a Salvinia molesta leaf [44]. Copyright (2010), WILEY-VCH Verlag GmbH \& Co. KGaA, Weinheim.

Fig. 6. Images of naturally superhydrophobic surfaces at different magnifications: (A) Morpho aega butterfly`s wing [45]. Copyright (2007), The Royal Society of Chemistry. (B) water strider`s leg [46]. Copyright (2004), Nature Publishing Group. (C) the back of Stenocara beetle [47]. Copyright (2001), Macmillan Magazines Ltd.

Fig. 7. Images of superhydrophobic surfaces in nature at different magnifications: (A) Shark skin [49]. Copyright (1999), Macmillan Magazines Ltd. (B) the gecko foot [52]. Copyright (2005), The National Academy of Sciences of the USA.

Fig. 8. SEM images of microstructures and the measurement of contact angle of the silicon wafers: (A) the exposure duration (Te) $=20 \mathrm{sec}$., contact angle $=125.8^{\circ}$, (B) the exposure duration $(\mathrm{Te})=30 \mathrm{sec}$., contact angle $(\mathrm{CA})=45^{\circ},(\mathrm{C})$ the exposure duration $(\mathrm{Te})=60 \mathrm{sec}$, the contact angle $=153.2^{\circ}[67]$. Copyright (2014), SPIE.

Fig. 9. Contact angles variations versus the cleanout times of hydrochloric acid for sample exposure durations of 20, 30, 60, and 90 sec., respectively [67]. Copyright (2014), SPIE. 
Fig. 10. (A) Superhydrophobic surface with roughness of micro- and nano-structures driven by the Marangoni flow. (B) SEM images at (a) $10 \%$, (b) $20 \%$, (c) $30 \%$, (d) $40 \%$, (e) $50 \%$, (f) $60 \%$, (g) $70 \%$, (h) $80 \%$, and (i) $(90 \%)$ ethanol showing the influence of ethanol concentration on preparation of superhydrophobic surface. The precursor solution involves trimethylpropane triacrylate (TMPTA) and ethanol. The inset images exhibit a static water droplet on each surface. (C) The value of the static water contact angle of each film [68]. Copyright (2014), American Chemical Society.

Fig. 11. (A) SEM images at 100x of the sanded aluminum surface: (a) aluminum surface etched by immersion in $\mathrm{CuCl} 2$ at 100x (c) and 5000x (e) magnifications, and the as-prepared surface etched via a combination of $\mathrm{CuCl}_{2}$ and a solution of $\mathrm{HCl}$ at 100x (b), 1000x (d), and 5000x (f) magnifications. (B) Water droplet contact angle on (a and b) plan bare aluminum surface with the oxide layer. (c and d)for the as-prepared super hydrophobic surface [75]. Copyright (2014), Elsevier.

Fig. 12. (A) micrographs of FESEM surfaces of (a) TF0 - untreated (b) TF2 - with 2 hours plasma treatment, (c) TF4 - with 4 hours plasma treatment, and (d) TF5 with 5 hours plasma treatment of PTFE samples. Insets illustrate the water droplet shape utilized for measurement of contact angles in terms of PTFE surfaces. (B) FESEM micrographs of PTFE sample, TF4 - with 4 hours plasma treatment, exhibiting the nature of bumps at magnifications (a) and (b) $30 \mathrm{kX}$, (c) $75 \mathrm{kX}$, and (d) $100 \mathrm{kX}$ [78]. Copyright (2014), Elsevier.

Fig. 13. (A) a 3D graphene foam network photograph, (B) SEM image of micro-porous graphene foam exhibiting an approximate pore size average of up to $200 \mu \mathrm{m}$. (C) Teflon-coated graphene foam exhibiting pores with the same dimensions and structures as the original graphene foam [83]. Copyright (2013), Wiley-VCH Verlag GmbH \& Co. KGaA, Weinheim. 
Fig. 14. (A) A diagram showing the preparation process for $\mathrm{PU} @ \mathrm{Fe}_{2} \mathrm{O}_{3} @ \mathrm{FB}$ sponges. (B) SEM images of (a) Polyurethane (PU), (b) PU@Fe32 4 @ $\mathrm{SiO}_{2}$, (c) PU@Fe3 $\mathrm{P}_{4} @ \mathrm{SiO}_{2} @ \mathrm{FB}$ (Chemical vapor deposition, CVD of TEOS for 24 h, 5\% FP solution) sponges [85]. Copyright (2015), American Chemical Society.

Fig. 15. (A) SEM image of a glass substrate was coated with silica film containing MeOH:MTMS fraction of 45:1 and ammonium hydroxide of $0.4 \mathrm{ml}$. (B) Image of water drop contact angle above the silica film containing MeOH:MTMS fraction of 45:1 and ammonium hydroxide of 0.4ml.[91] Copyright (2013), Trans Tech Publications, Switzerland (C) The molecular structures of (a) $\mathrm{SiF}$ and (b) $\mathrm{SiP}$ representing the sol-gel precursors [92]. Copyright (2014), Springer Science+Business Media New York.(D) the total properties of the silica-coated microscope substrate [94]. Copyright (2014), Elsevier.

Fig. 16. SEM images of superhydrophobic surfaces fabricated by different electrochemical deposition strategies: (a) The Ni-Co alloy coating by two-step electrodeposition method [101]. Copyright (2015), Elsevier. (b) Co coatings fabricated by two-step electrochemical deposition method showing fluffy shell structures [102]. Copyright (2015), Elsevier. (c) A zinc deposit after modification with polypropylene (PP) [103]. Copyright (2014), Elsevier. (d) Cu mesh wires of copper nanoparticle (CU NPs)-coated Cu mesh film. [104]. Copyright (2013), AIP Publishing LLC.

Fig. 17. (a) SEM image of electrospun composite film with a weight ratio of $\mathrm{Fe}_{3} \mathrm{O}_{4} @ \mathrm{SiO}_{2} @ \mathrm{POTS} / \mathrm{PVDF}$ : 0.15:12. The inset exhibits the behavior of a water droplet on its surface. $\mathrm{CA}=150.3 \pm 0.3^{\circ}$. (b) Magnified view of this composite film (c). Variation of contact angle (CA) value of composite films depending on different weight ratios of $\mathrm{Fe}_{3} \mathrm{O}_{4} @ \mathrm{SiO}_{2} @ \mathrm{POTS} / \mathrm{PVDF}:$ (a) 0.10:12, $\mathrm{CA}=148.2 \pm 0.4^{\circ}$, (b) 0.12:12, CA = 149.1 $\pm 0.3^{\circ}$, (c) $0.15: 12, \mathrm{CA}=150.3 \pm 0.3^{\circ}$, (d) $0.20: 12, \mathrm{CA}=151.4 \pm 0.5^{\circ}$, and (e) $0.30: 12, \mathrm{CA}=152.4 \pm$ $0.4^{\circ}$.[111]. Copyright (2013), Elsevier. (d) SEM image of a zein cast film: ZEF made from a 
solution with $10 \mathrm{wt} \%$ of zein in $80 \%$ ethanol. (e) WCA image of a ZEF made from a solution with 10 wt \% of zein in 80\% ethanol [114]. Copyright (2015), American Chemical Society.

Fig. 18. (A) Photographs of water droplets on untreated cotton fabric and superhydrophobic cotton fabric coated with 5 layers of HMBS@LDH (B) SEM images of (a) untreated cotton fiber, and (b-d) cotton fibers coated with (b) 1, (c) 3, and (d) 5 layers of HMBS@LDH [118]. Copyright (2013), Elsevier.

Fig. 19. (a) SEM images of the glass slide with deposited candle soot; (b) the prepared $\mathrm{TiO}_{2}$ coating after the nanoimprint and calcination approach ; (c) schematic diagram of the fabrication procedure of the superhydrophobic film [123]. Copyright (2013), the Royal Society of Chemistry.

Fig. 20. (a) Cylindrical posts fabricated by utilizing a InVision 3-D printer measuring 175 micron diameter x1300 microns tall, and (b) a 2mm diameter water droplet posed atop a similar surface which demonstrate superhydrophobic behavior [128]. Copyright (2013), Inderscience Enterprises Ltd.

Fig. 21. Superhydrophobic iLattice and hollow iBall. (A) SEM images of iLattice and (C) iBall and (B) dyed water on poly(PFMA)-iLattice and (D) filled poly(PFMA)-iBall [130]. Copyright (2013), The Royal Society of Chemistry.

Fig. 22 Side view SEM image of silicon surfaces that textured by $800 \mathrm{~nm}, 10$ fs irradiation at various lase fluences. (a) $0.17 \mathrm{~J} / \mathrm{cm}_{2}$, (b) $0.56 \mathrm{~J} / \mathrm{cm}_{2}$, (c) $1.35 \mathrm{~J} / \mathrm{cm}_{2}$, and (d) $2.25 \mathrm{~J} / \mathrm{cm}_{2}$ (scale bar 
$5 \mu \mathrm{m})$. Higher magnifications of the obtained structures are shown in the corresponding insets (scale bar $1 \mu \mathrm{m}$ ) [134]. Copyright (2008), Springer-Verlag.

Fig. 23 Static contact angle $(\bullet)$ and sliding angle (०) measurements of a water droplet on structured silicon surfaces plotted as a function of the laser fluence. Regarding fluences below $0.5 \mathrm{~J} / \mathrm{cm}^{2}$, the sliding angles are greater than the $30^{\circ}$. The lines are guides for the eye. Images of the droplet on the flat as well as on the structured silicon surface are shown at the insets [134]. Copyright (2008), Springer-Verlag.

Fig. 24. The effect of substrate temperature of uncoated and HDFT-coated Ag nanorod substrates on the contact angle [146]. Copyright (2014), AIP Publishing LLC.

Fig. 25. (A) SEM images of Ti substrate showing the applied anodization voltages on the morphology of the surface at (a) $20 \mathrm{~V}$, (b) $40 \mathrm{~V}$, (c) $60 \mathrm{~V}$, and (d) $80 \mathrm{~V}$; (B) and (C) exhibits the effect of electrolyte temperature and anodization voltage on the contact angle value, respectively [151]. Copyright (2015), Elsevier. 


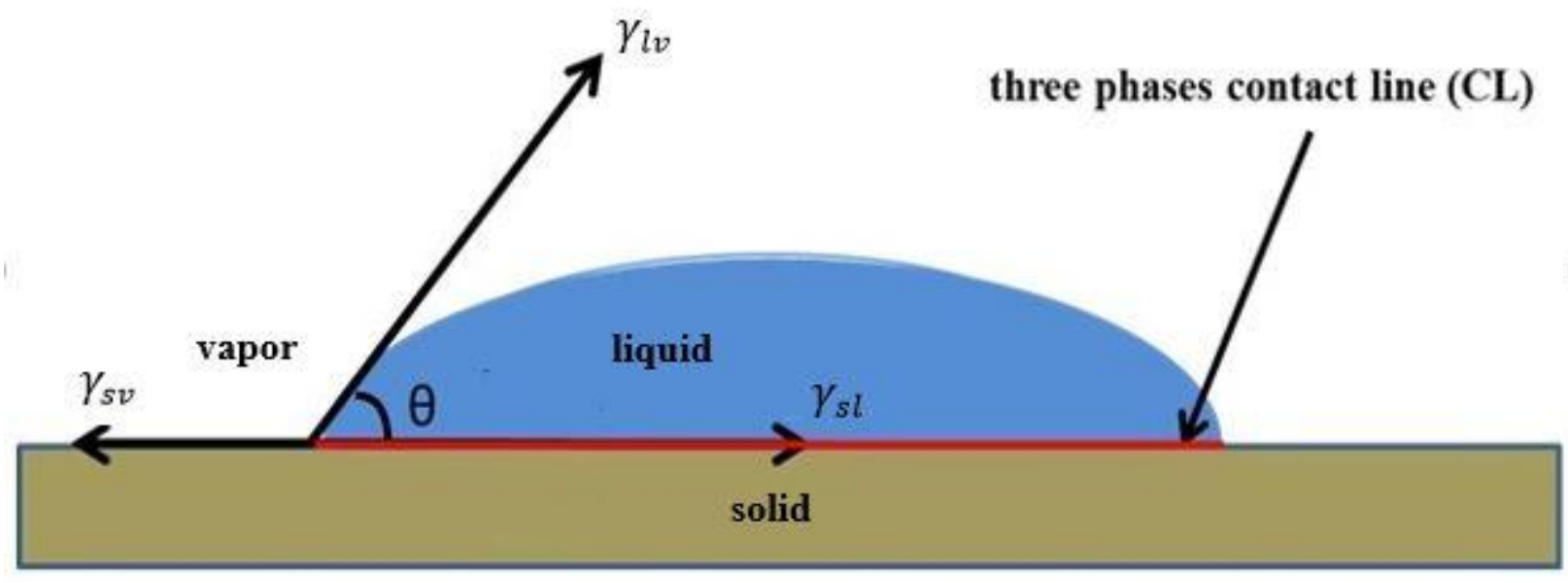

Fig. 1

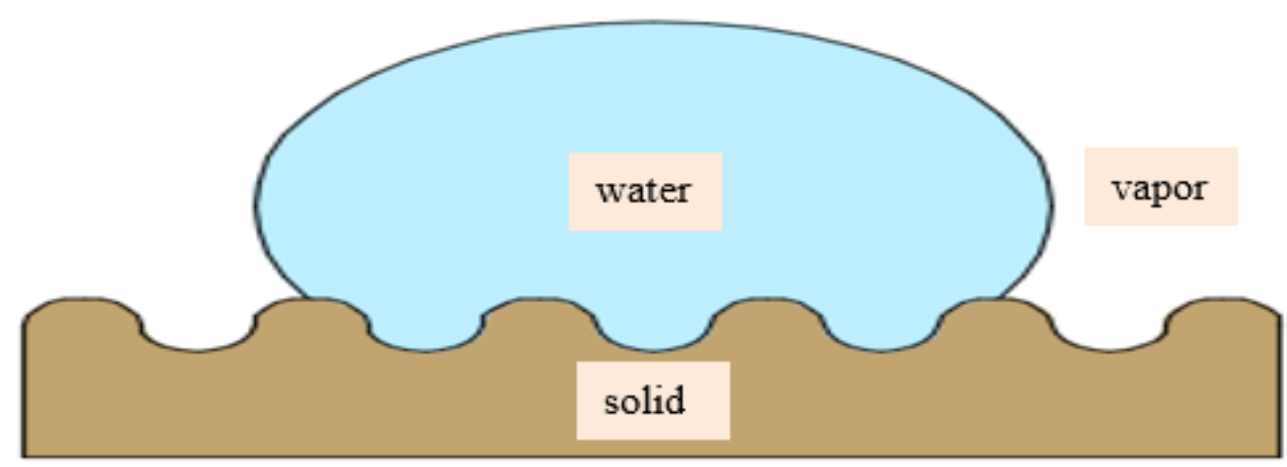

Fig. 2 


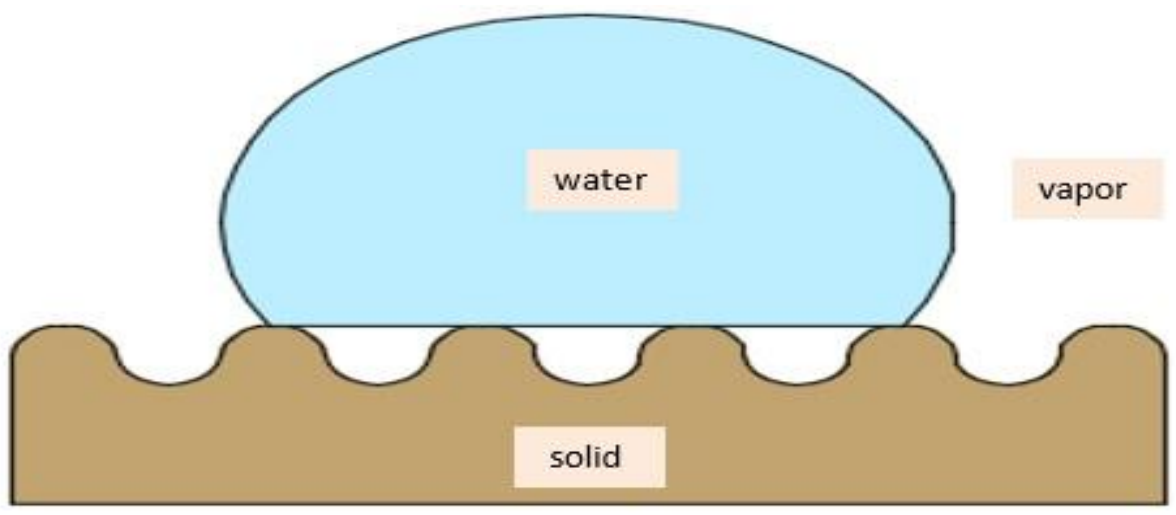

Fig. 3

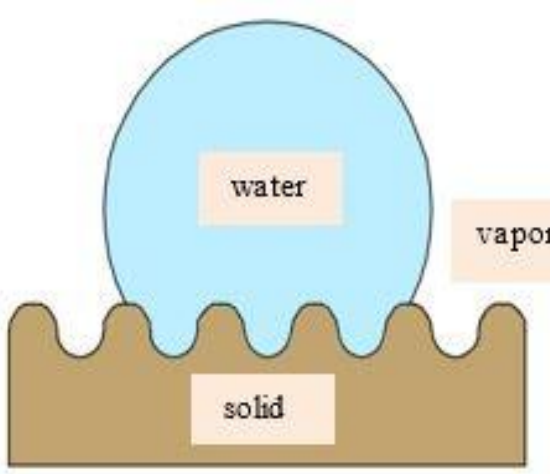

Wenzel's mode

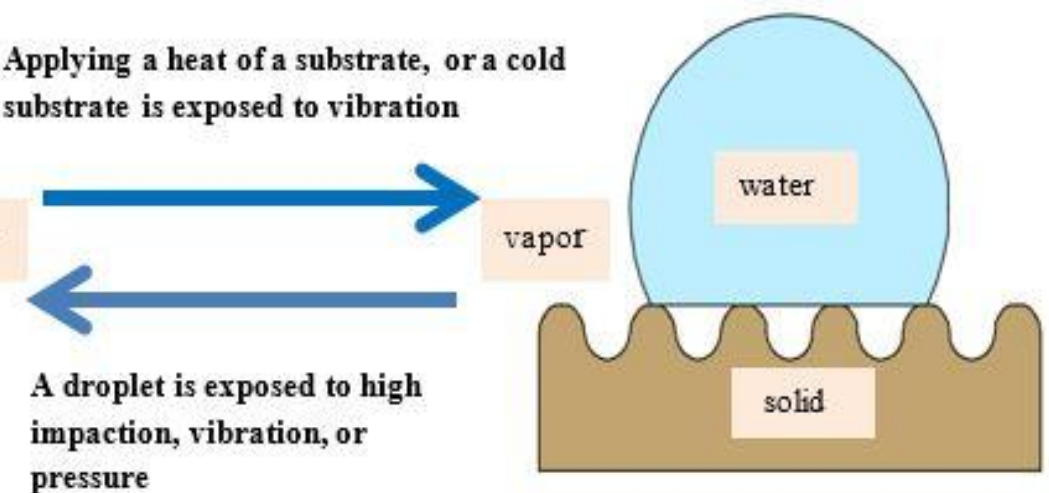

Cassie's mode

Fig. 4 
A
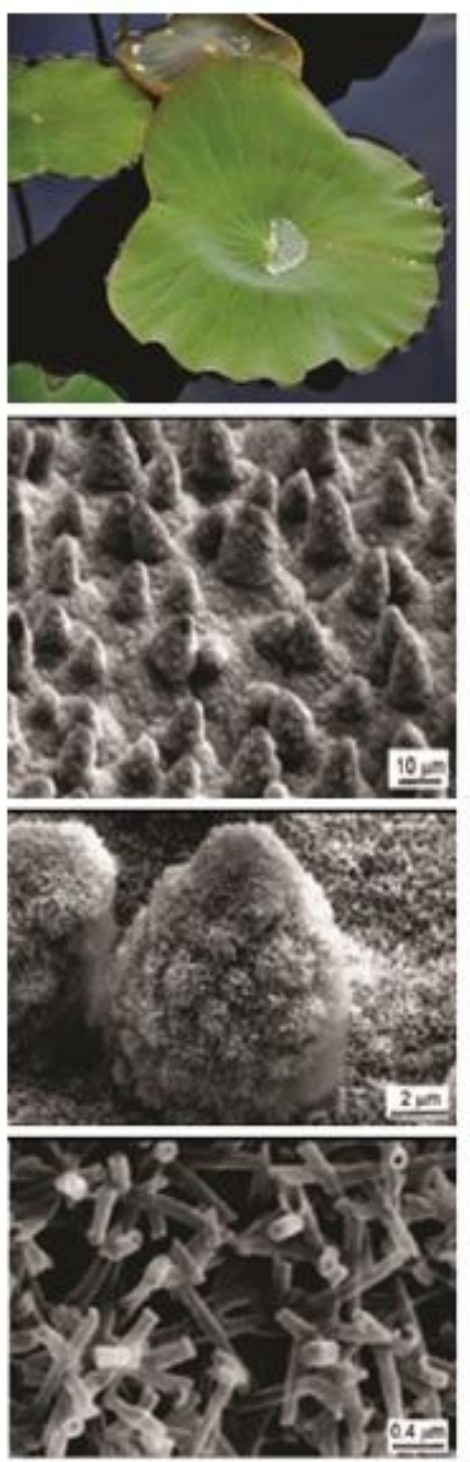

B
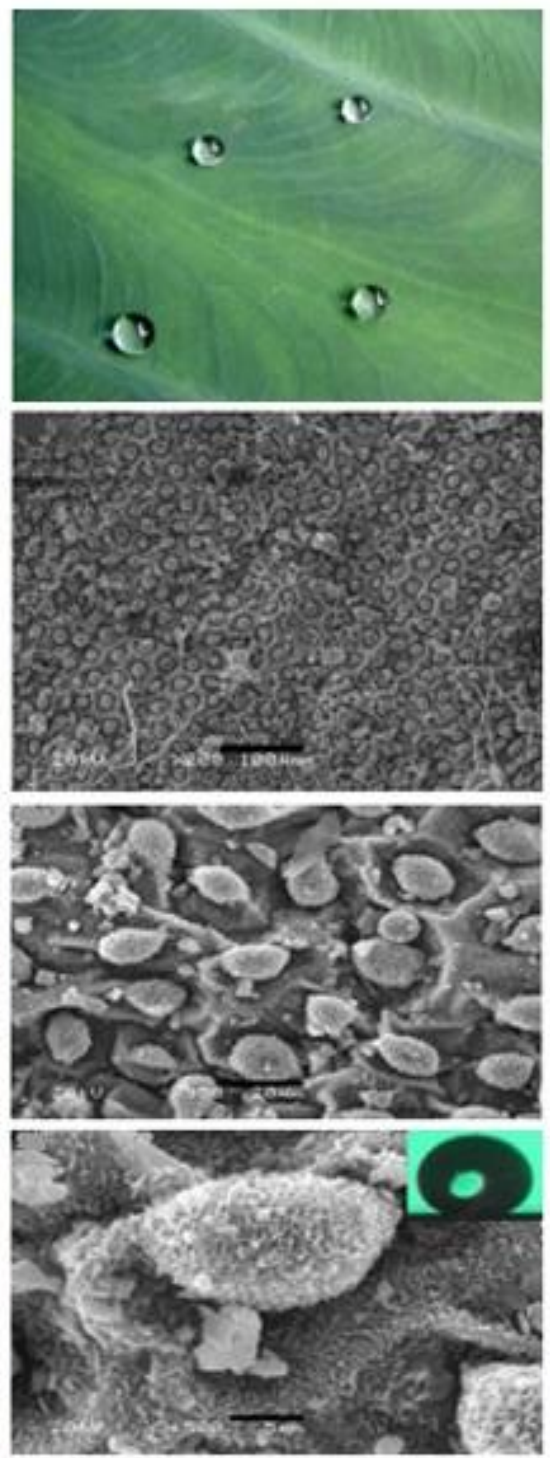

C
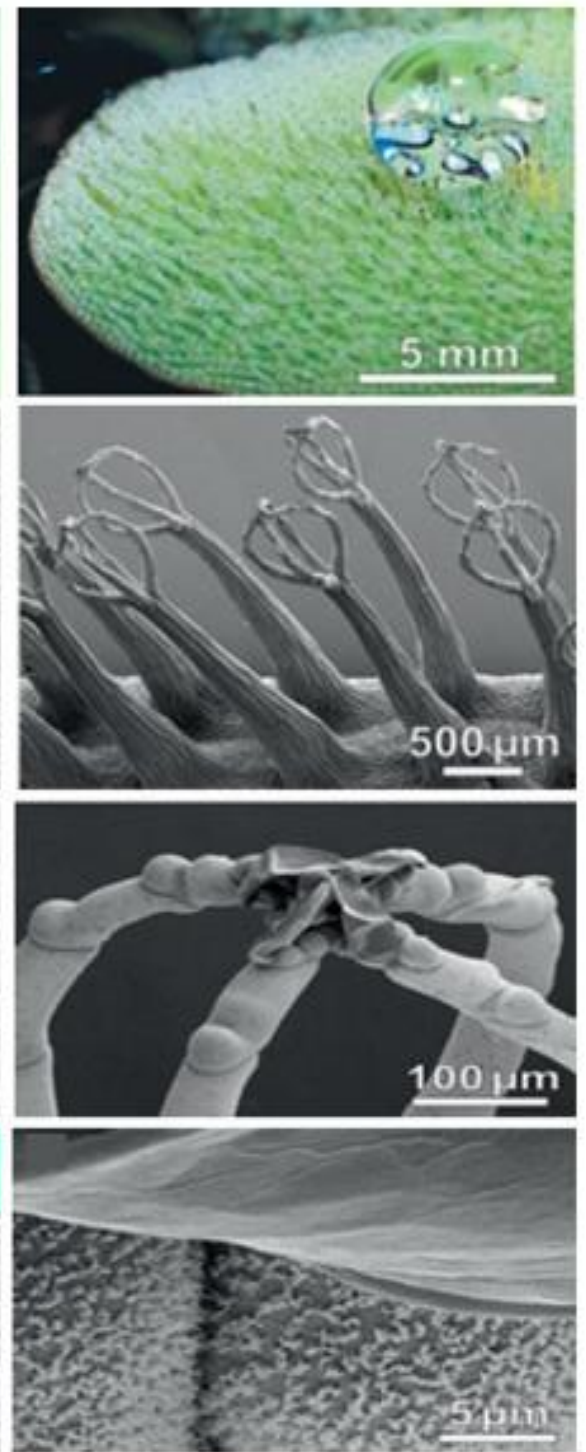

Fig. 5 
A
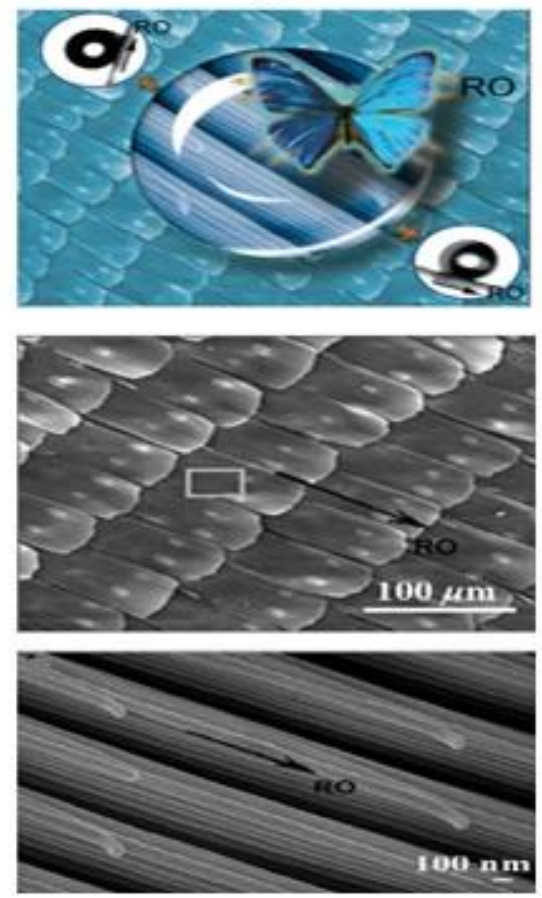

B
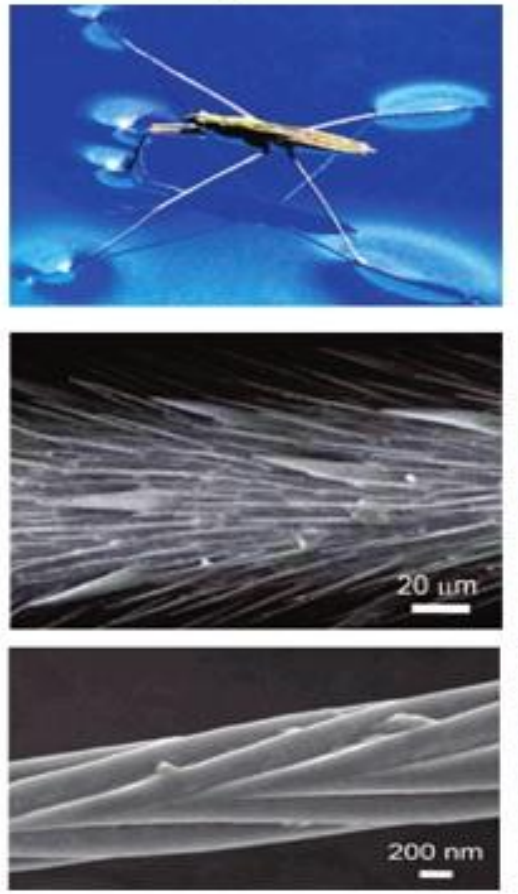

C

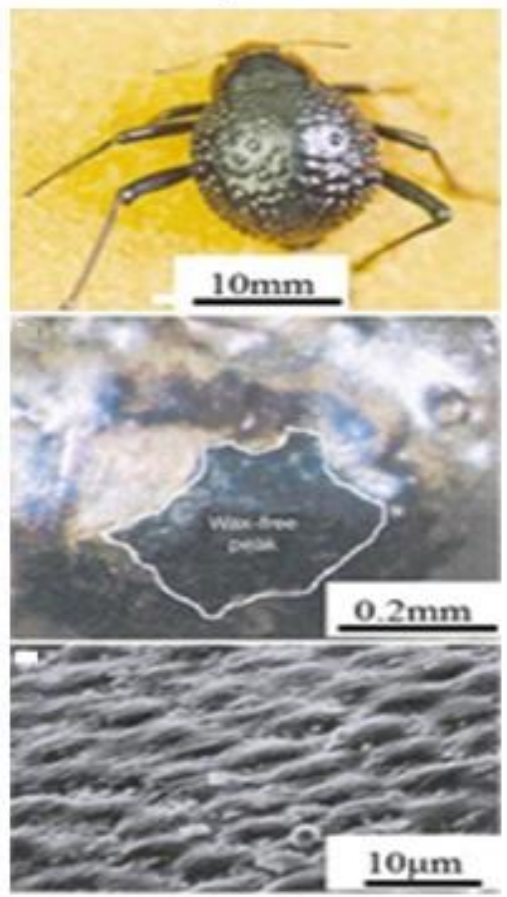

Fig. 6

A

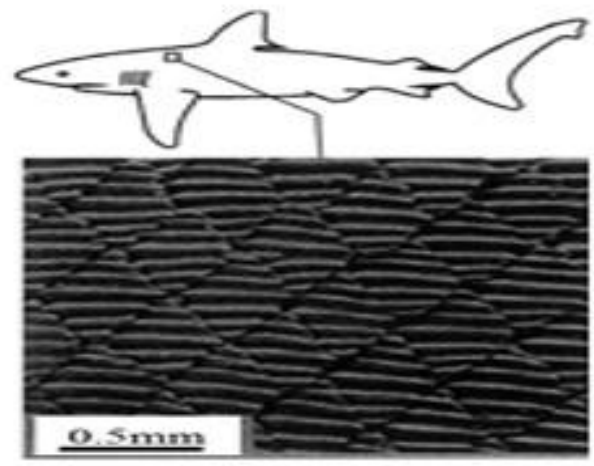

B

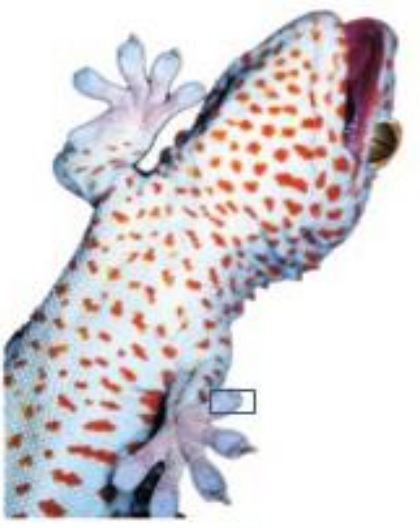

Fig. 7 


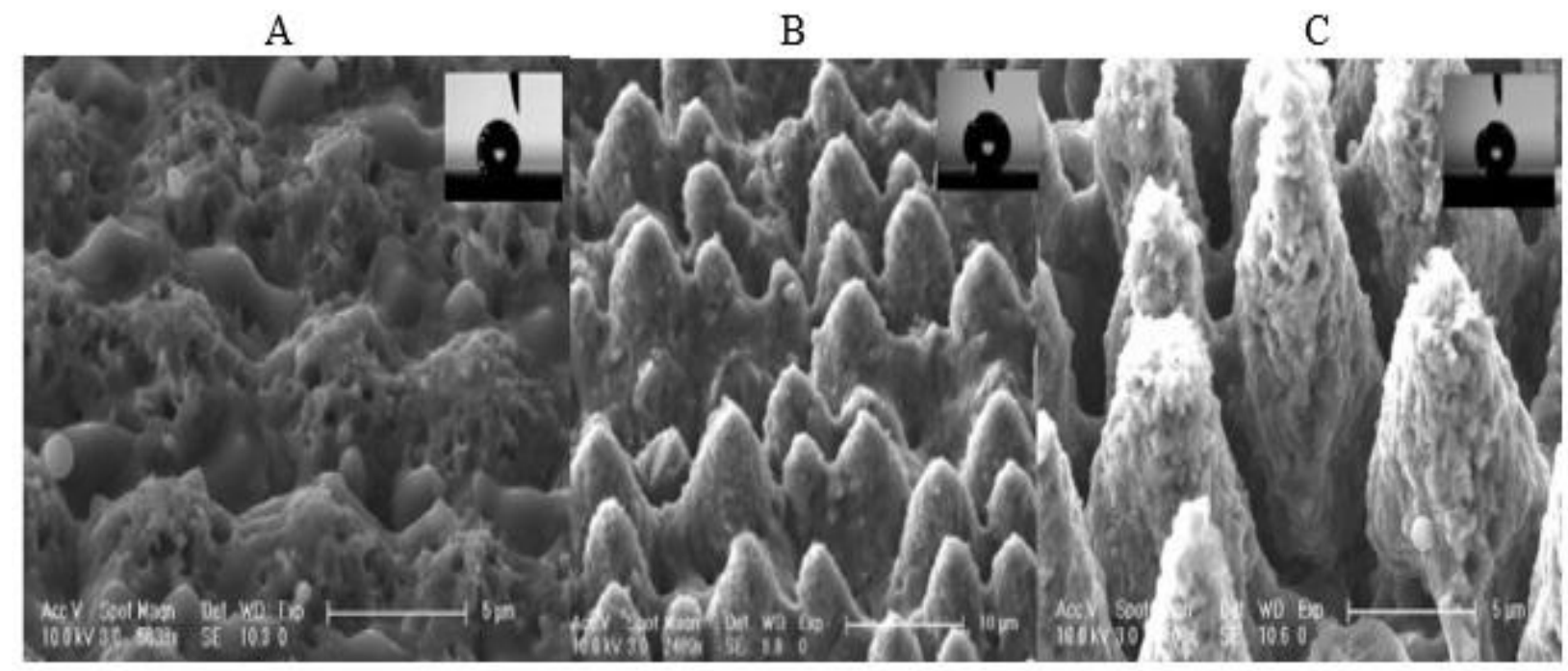

Fig. 8

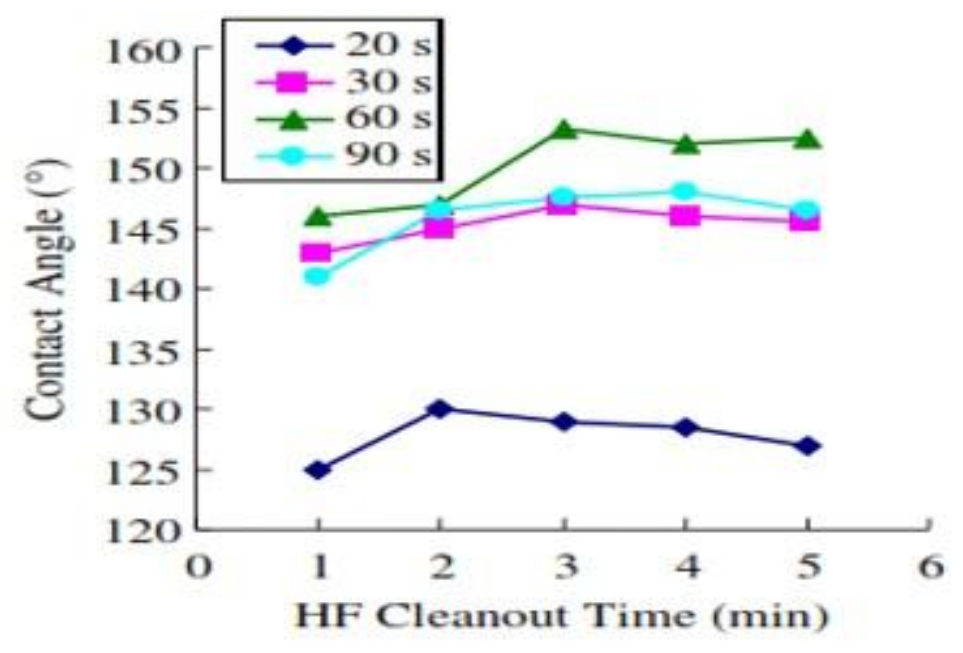

Fig. 9 

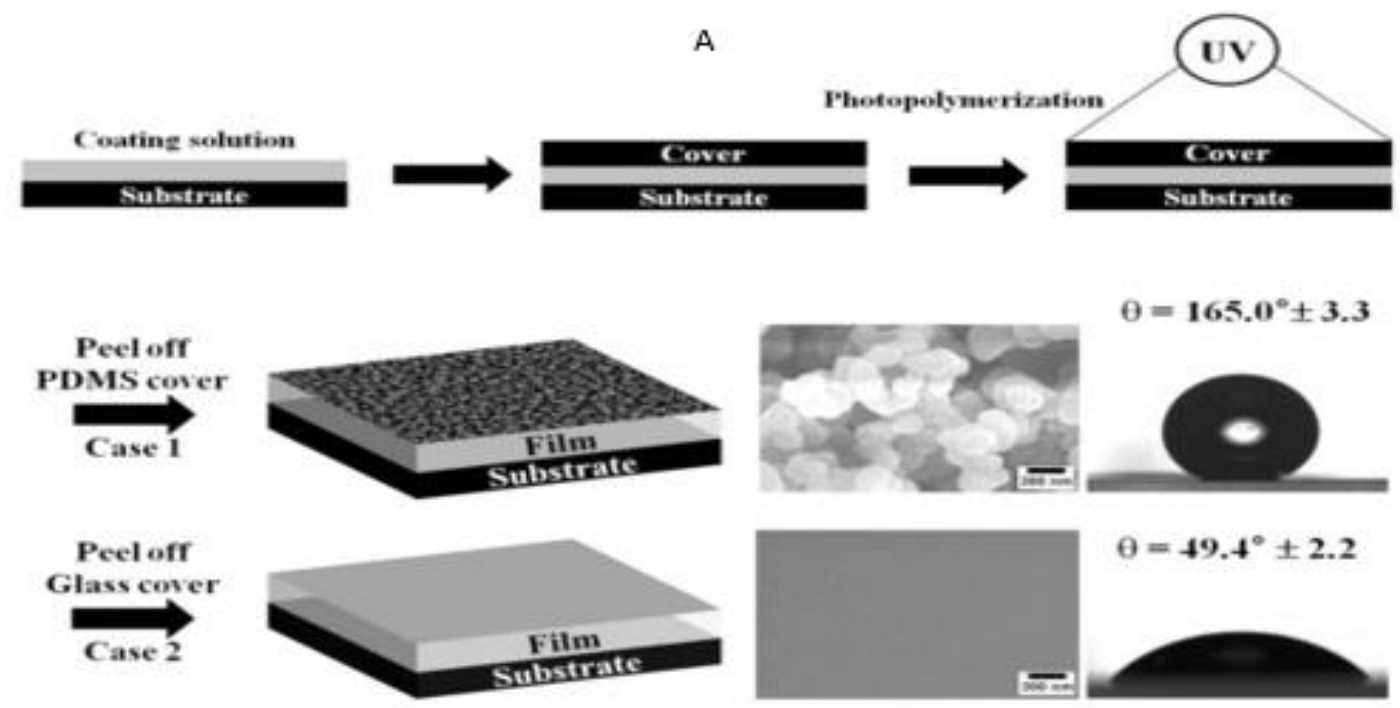

$$
\theta=49.4^{\circ} \pm 2.2
$$

B

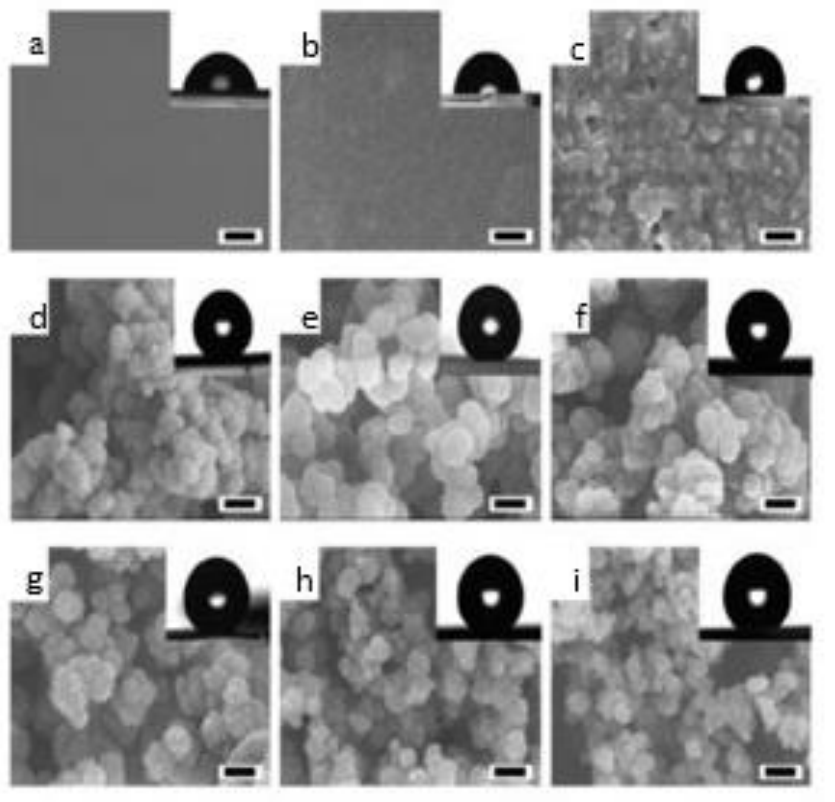

C

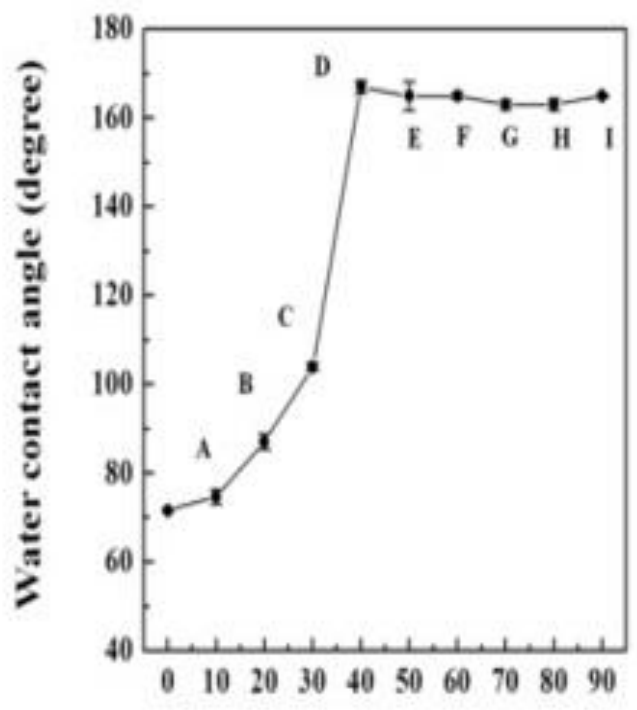

Ethanol concentration (\%)

Fig. 10 
A

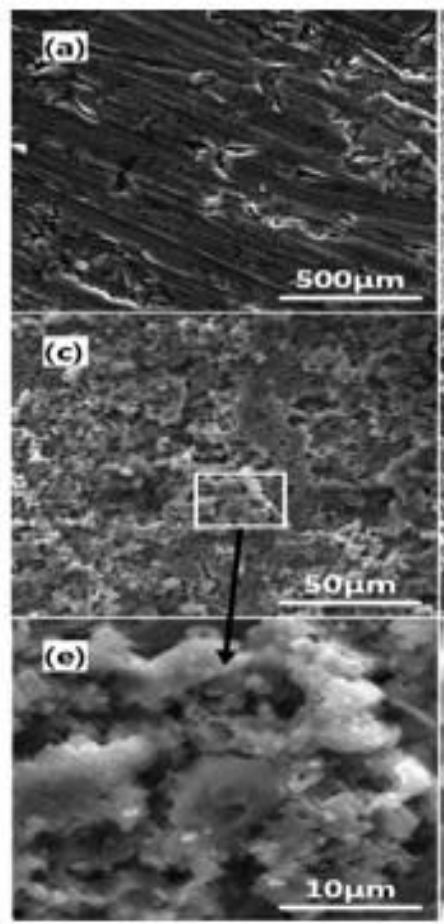

B

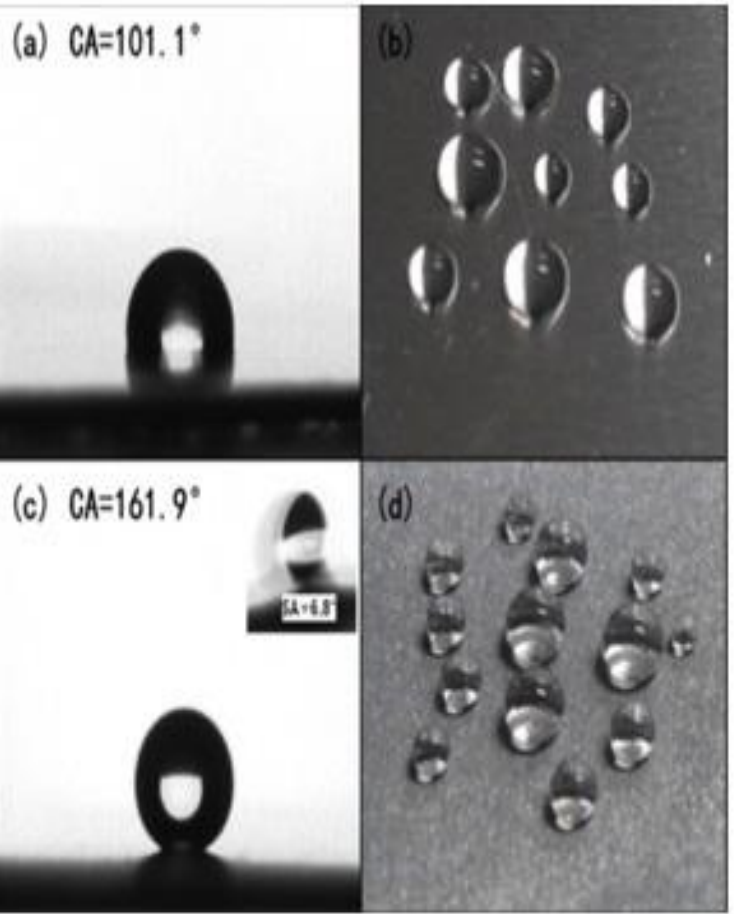

Fig. 11

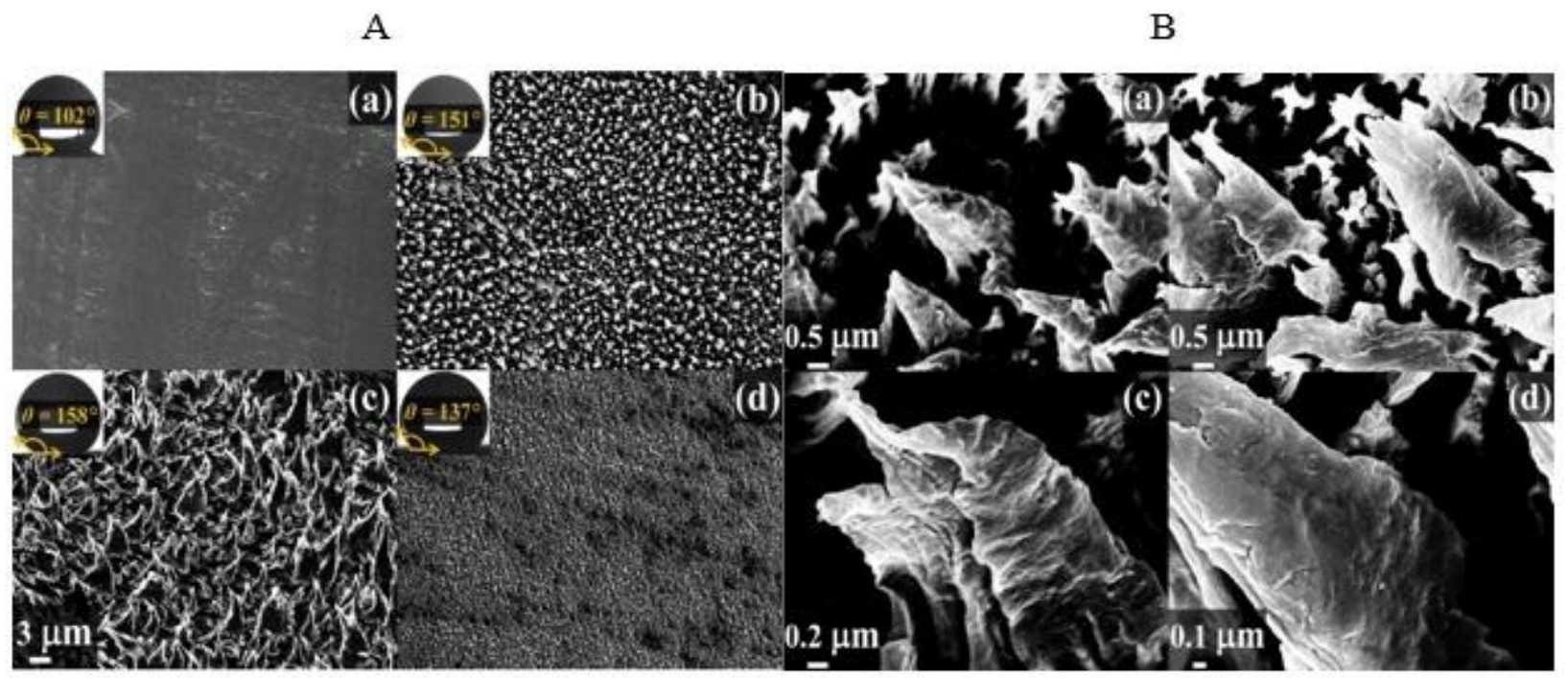

Fig. 12 
A

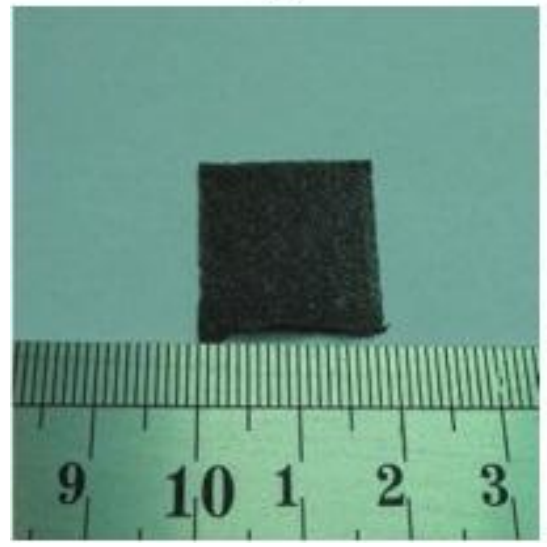

C

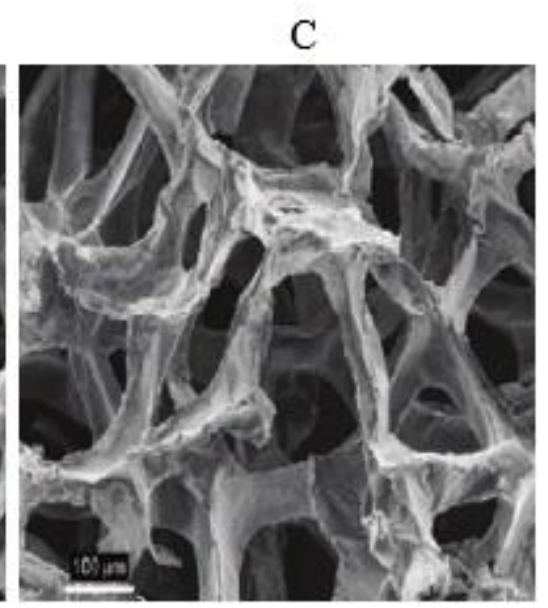

Fig. 13

A
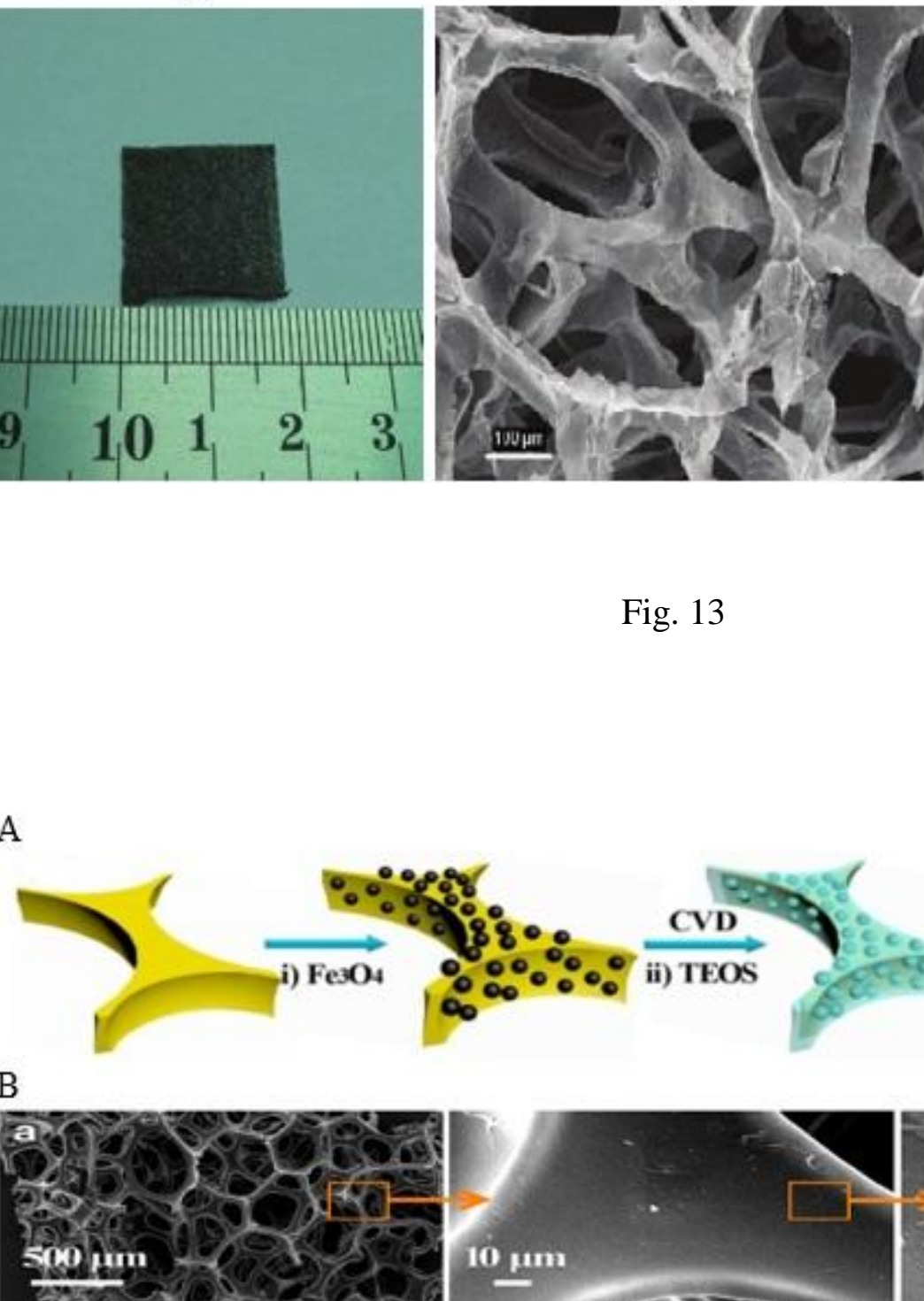

Fig. 13 


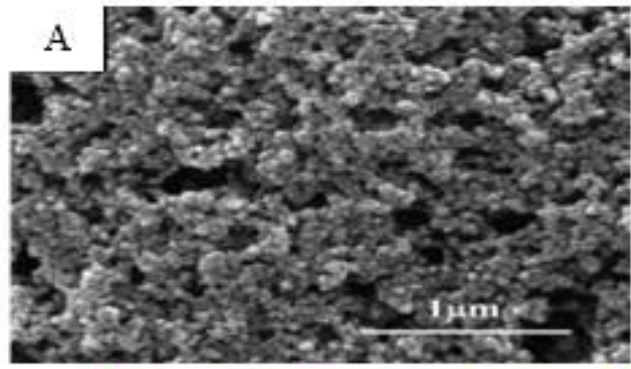

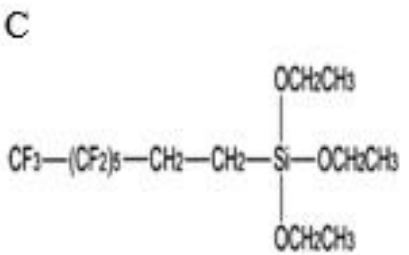

(a)<smiles>CO[Si](C)(O)CCCNP(=O)(c1ccccc1)c1ccccc1</smiles>

(b)

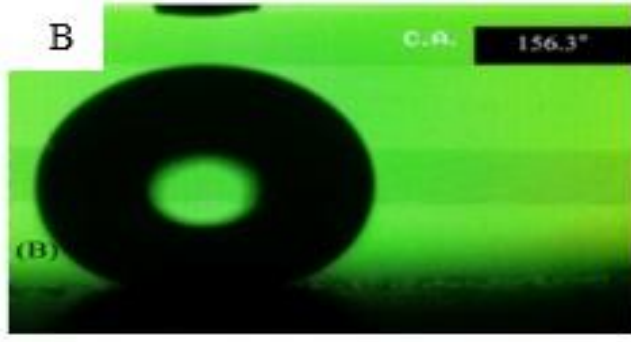

\section{$\mathrm{D}$}

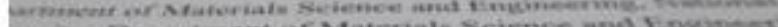

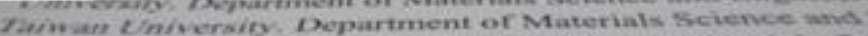
frrionaf Rawan Einiversify. Department of Materials Sf vincerion bal Taiwan University- Departenent

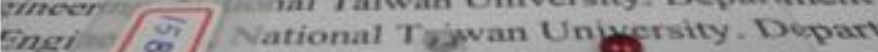

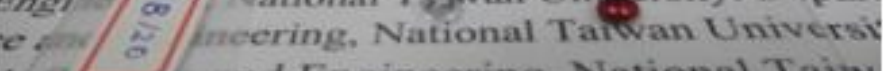

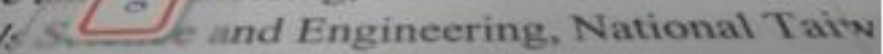
nf of Materials Science and Engineerin.

Fig. 15
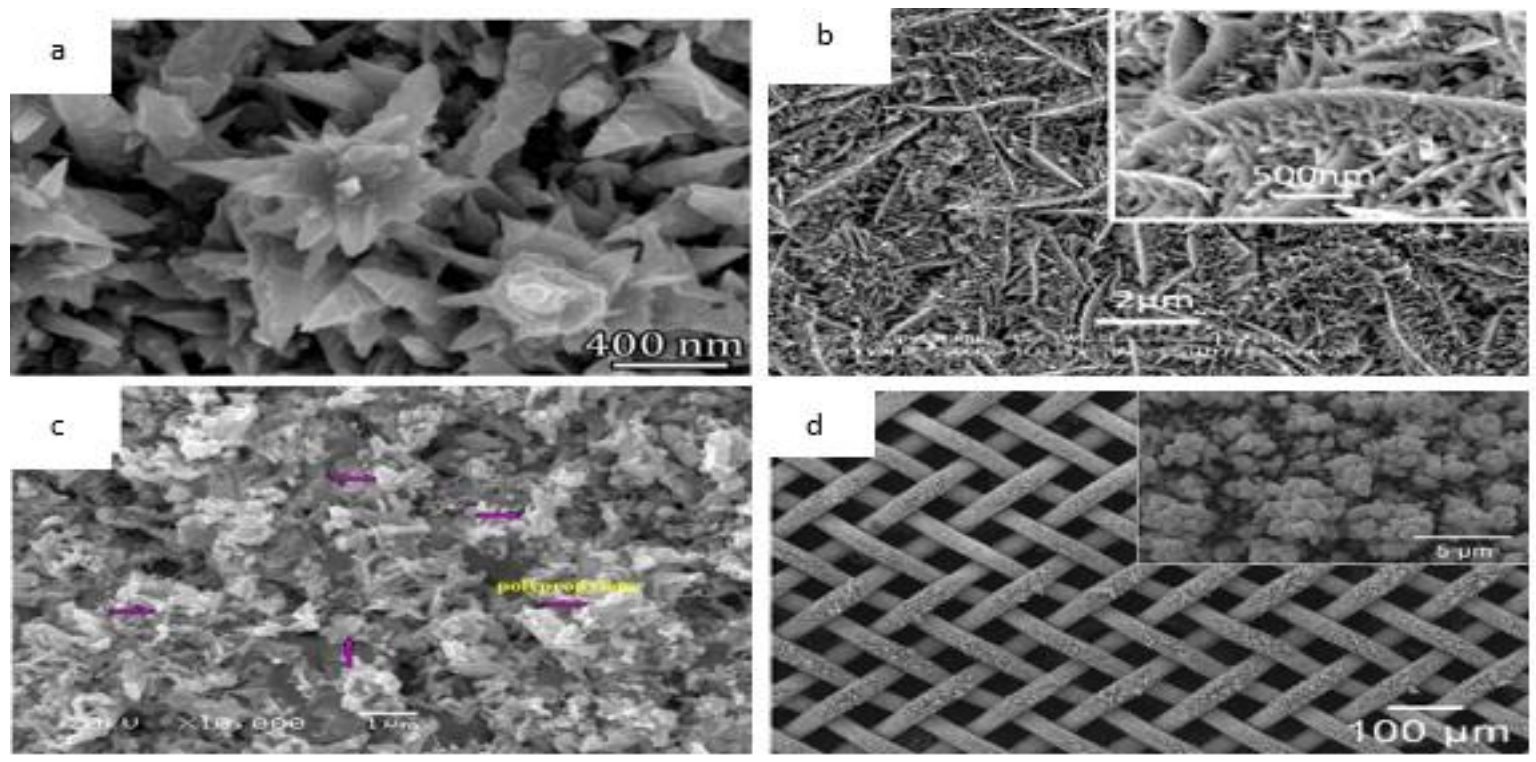

Fig. 16 

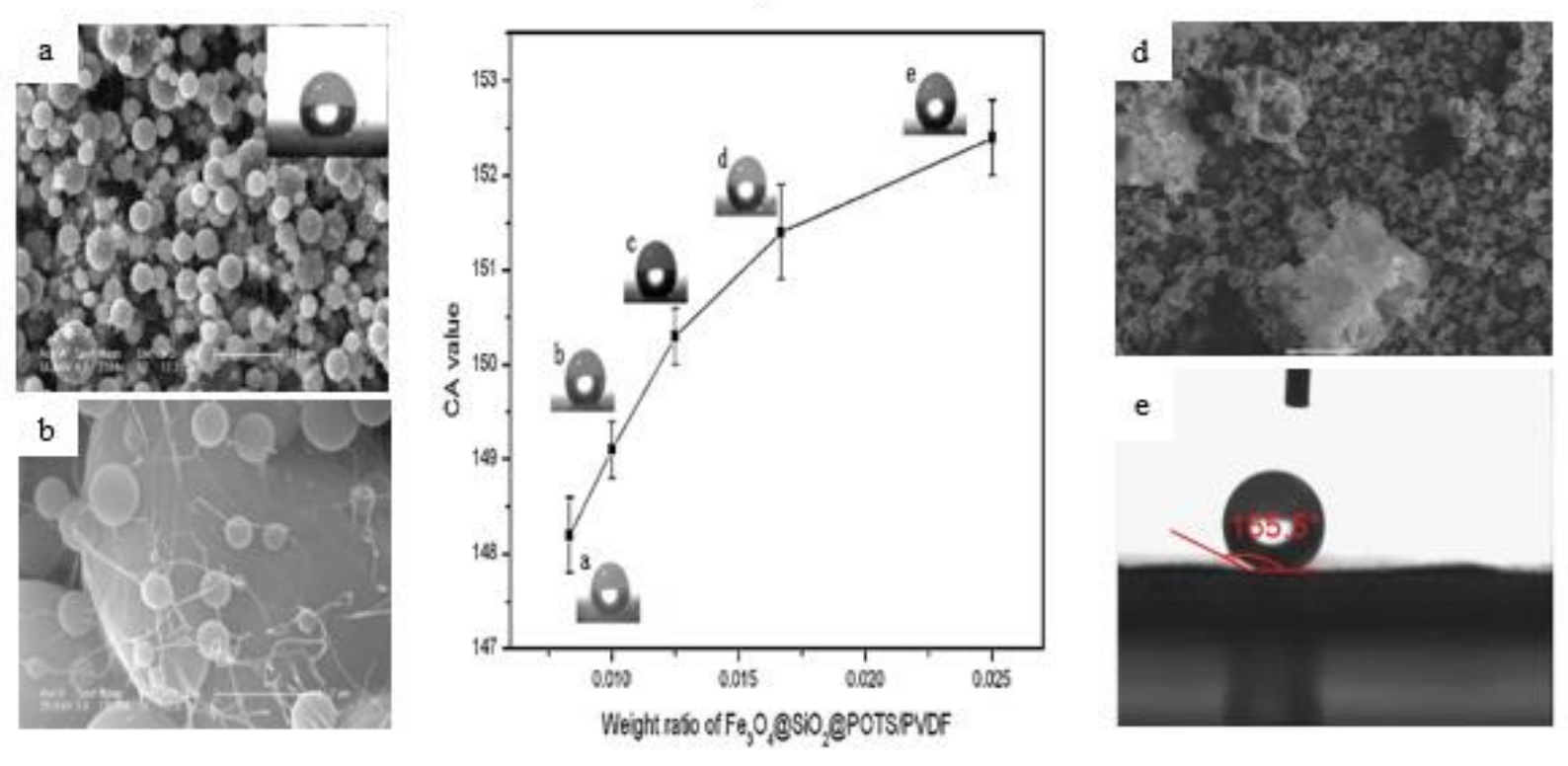

Fig. 17
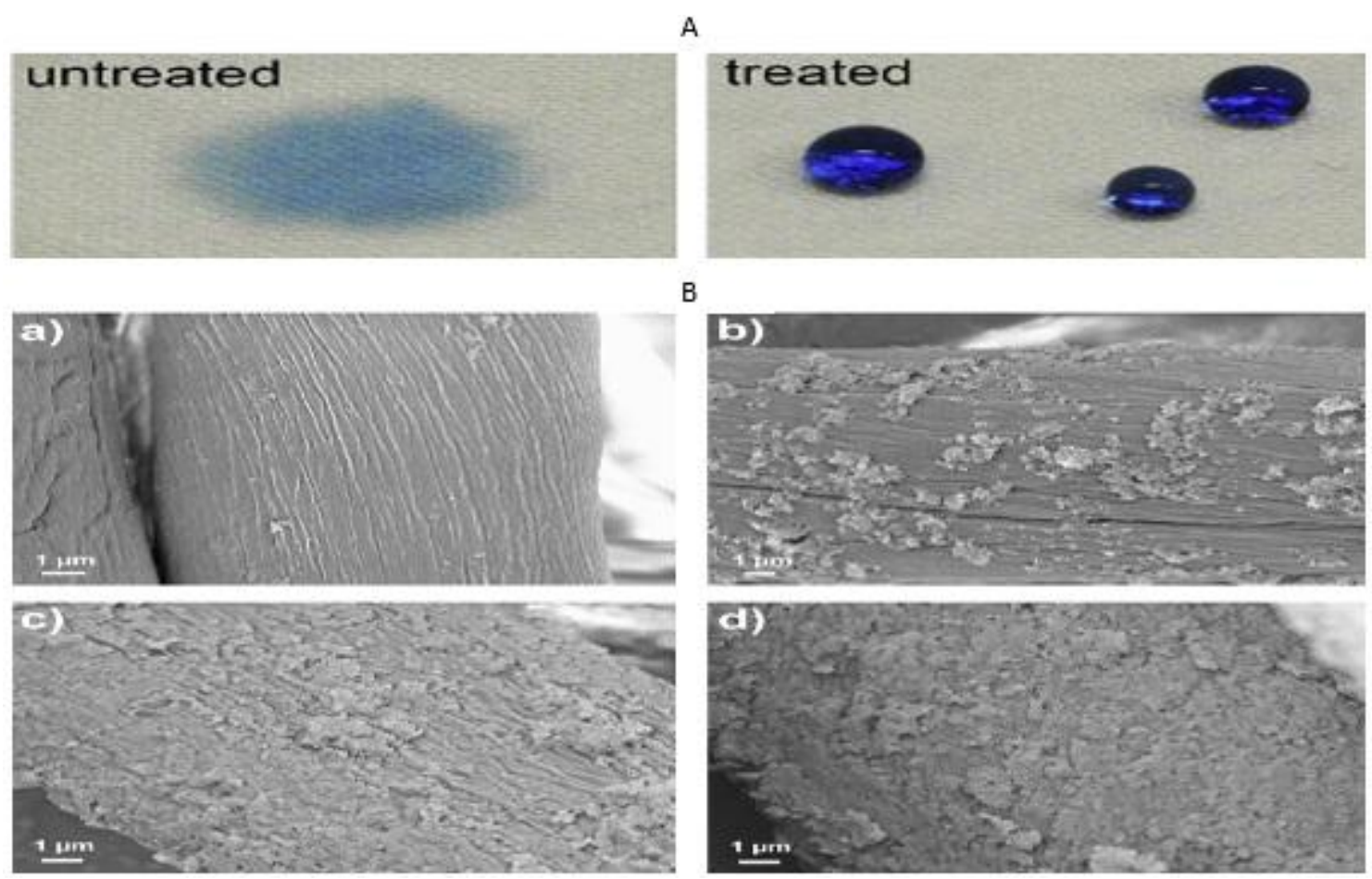

Fig. 18 

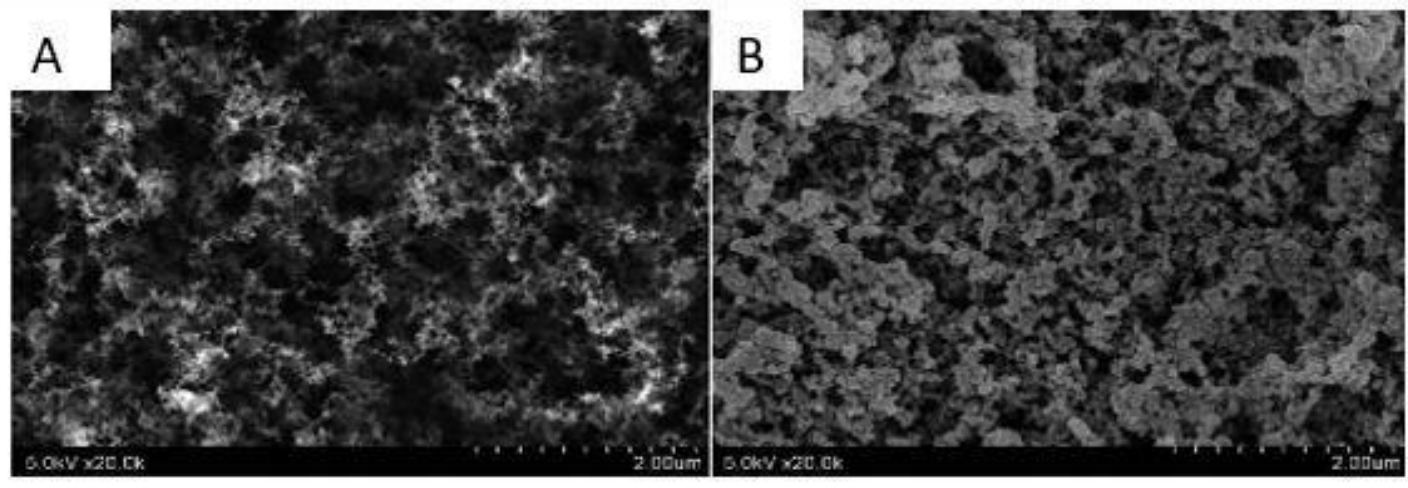

C

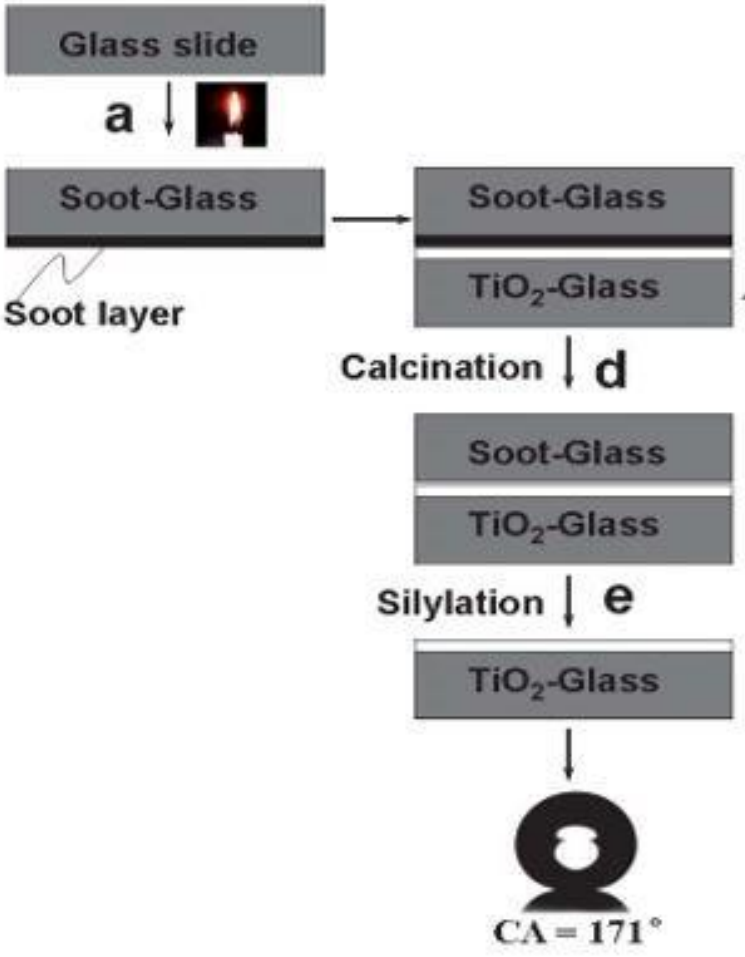

Fig. 19 

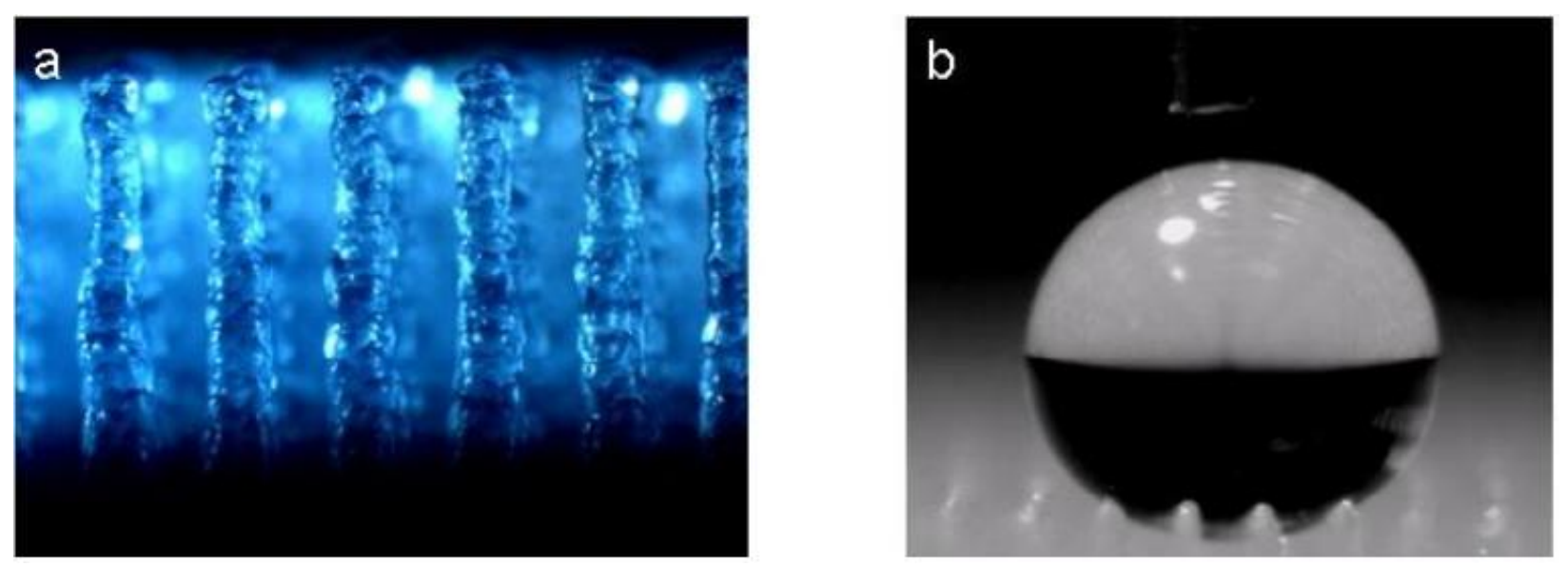

Fig. 20
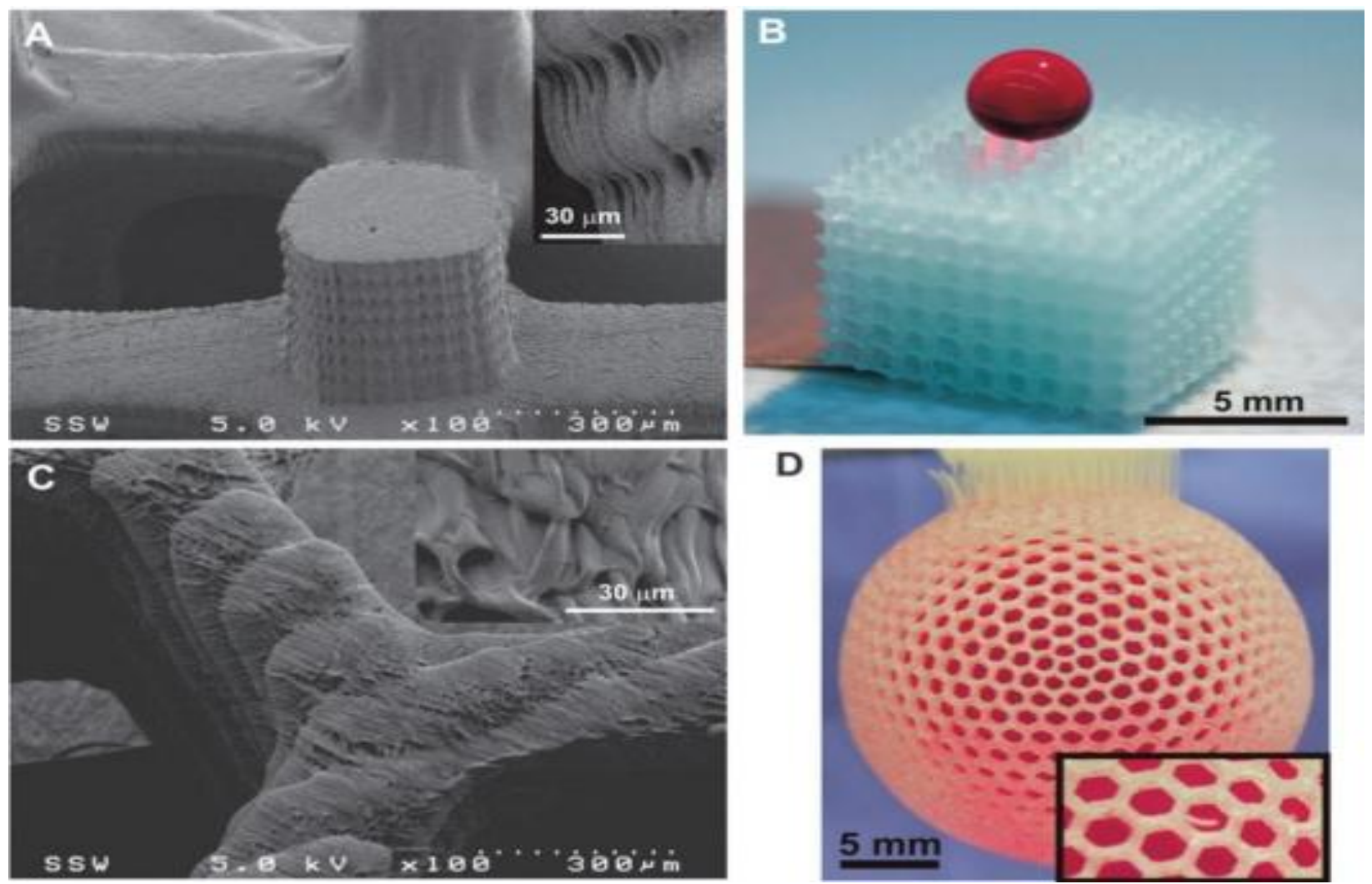

D

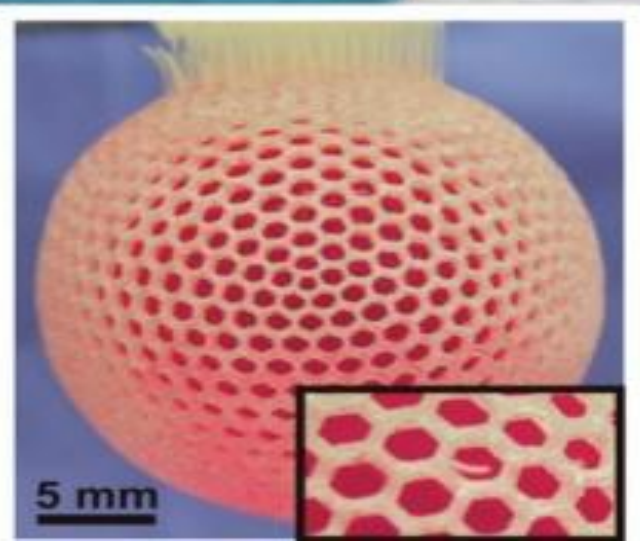

Fig. 21 

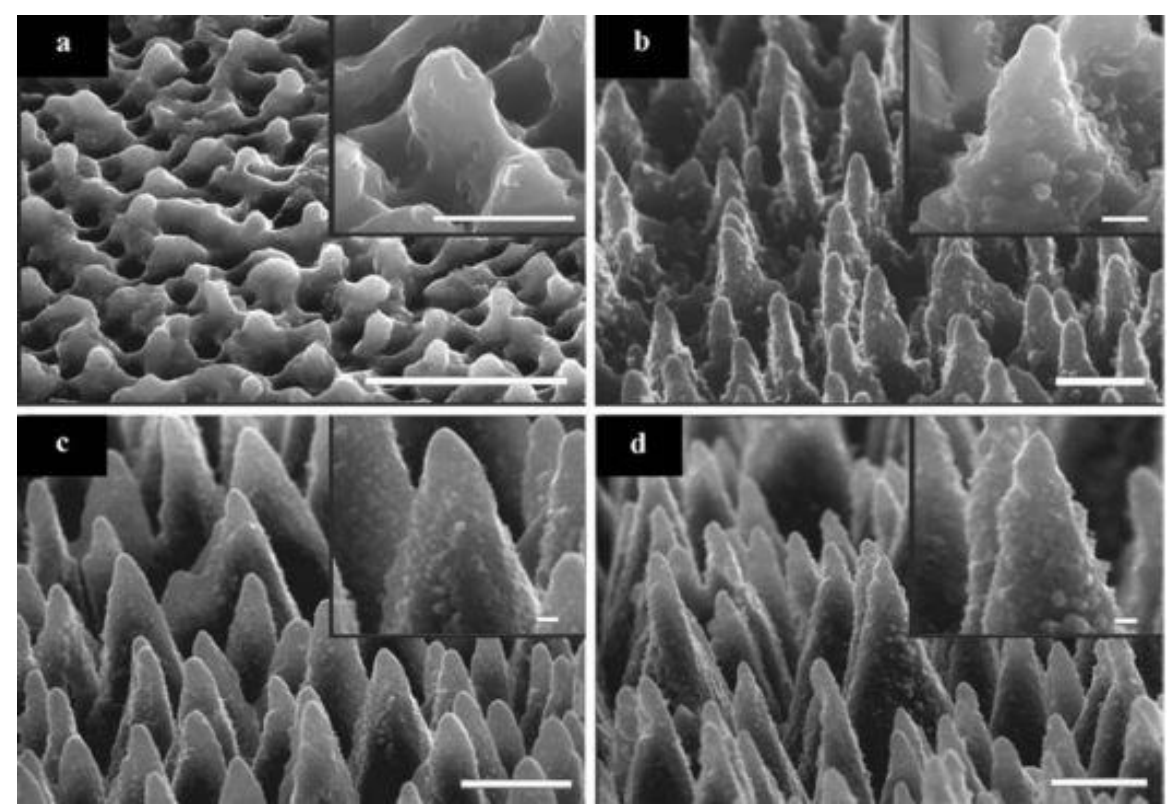

Fig. 22

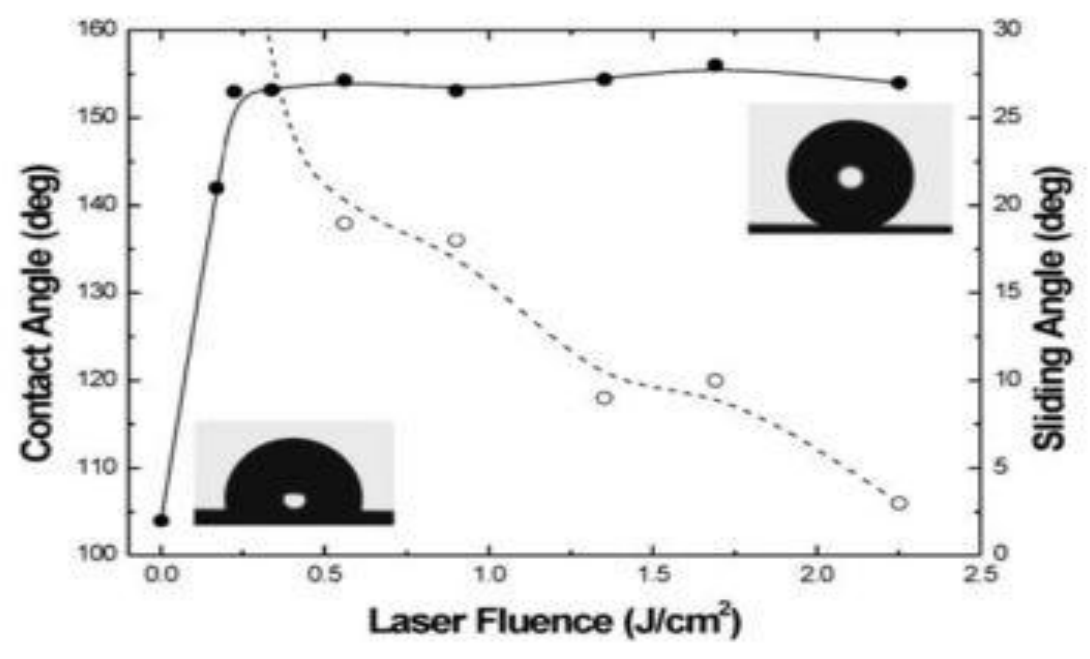

Fig. 23 


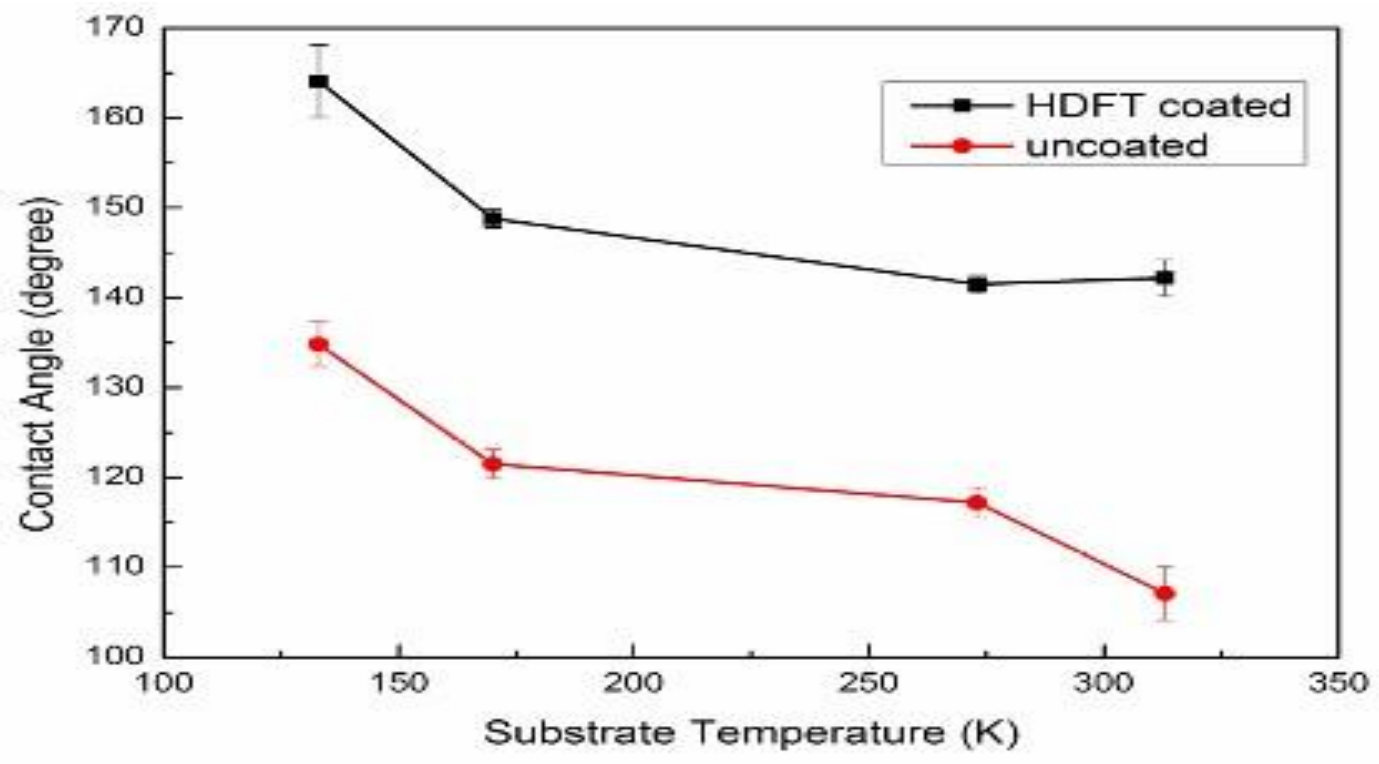

Fig. 24
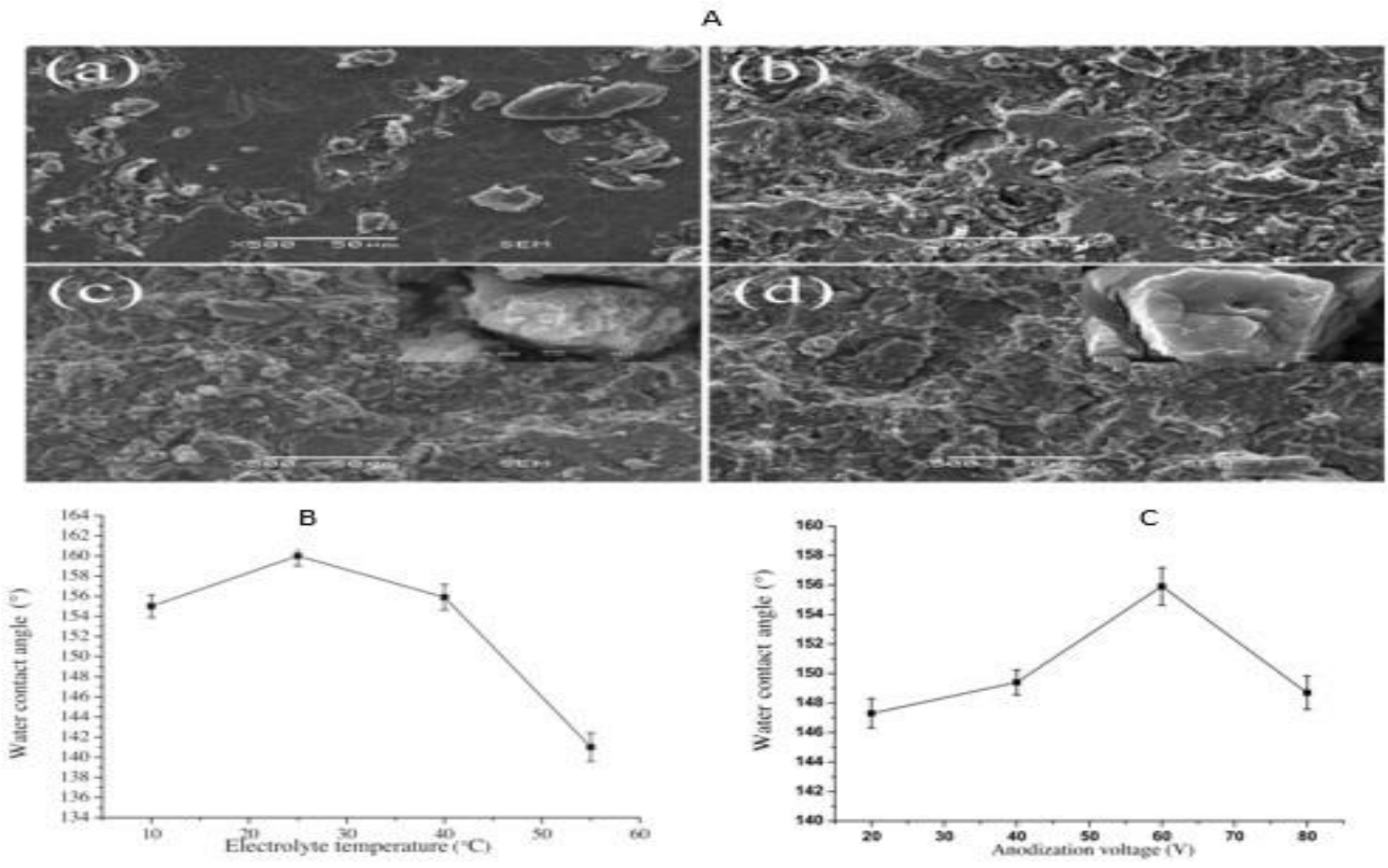

Fig. 25 